\title{
Genome-wide association study of agronomic traits in bread wheat reveals novel putative alleles for future breeding programs
}

\author{
Yousef Rahimi ${ }^{1,3}$, Mohammad Reza Bihamta ${ }^{1 *}$, Alireza Taleei ${ }^{1}$, Hadi Alipour ${ }^{2}$ and Pär K. Ingvarsson ${ }^{3}$
}

\begin{abstract}
Background: Identification of loci for agronomic traits and characterization of their genetic architecture are crucial in marker-assisted selection (MAS). Genome-wide association studies (GWAS) have increasingly been used as potent tools in identifying marker-trait associations (MTAs). The introduction of new adaptive alleles in the diverse genetic backgrounds may help to improve grain yield of old or newly developed varieties of wheat to balance supply and demand throughout the world. Landraces collected from different climate zones can be an invaluable resource for such adaptive alleles.
\end{abstract}

Results: GWAS was performed using a collection of 298 Iranian bread wheat varieties and landraces to explore the genetic basis of agronomic traits during 2016-2018 cropping seasons under normal (well-watered) and stressed (rain-fed) conditions. A high-quality genotyping by sequencing (GBS) dataset was obtained using either all original single nucleotide polymorphism (SNP, 10938 SNPs) or with additional imputation (46,862 SNPs) based on W7984 reference genome. The results confirm that the $\mathrm{B}$ genome carries the highest number of significant marker pairs in both varieties (49,880, 27.37\%) and landraces (55,086, 28.99\%). The strongest linkage disequilibrium (LD) between pairs of markers was observed on chromosome 2D (0.296). LD decay was lower in the D genome, compared to the A and B genomes. Association mapping under two tested environments yielded a total of 313 and 394 significant $\left(-\log _{10} P>3\right)$ MTAs for the original and imputed SNP data sets, respectively. Gene ontology results showed that 27 and $27.5 \%$ of MTAs of SNPs in the original set were located in protein-coding regions for well-watered and rain-fed conditions, respectively. While, for the imputed data set 22.6 and $16.6 \%$ of MTAs represented in protein-coding genes for the well-watered and rain-fed conditions, respectively.

Conclusions: Our finding suggests that Iranian bread wheat landraces harbor valuable alleles that are adaptive under drought stress conditions. MTAs located within coding genes can be utilized in genome-based breeding of new wheat varieties. Although imputation of missing data increased the number of MTAs, the fraction of these MTAs located in coding genes were decreased across the different sub-genomes.

Keywords: Wheat, Agronomic traits, GWAS, MTAs, Gene annotation

\footnotetext{
*Correspondence: mrghanad@ut.ac.ir

'Department of Agronomy and Plant Breeding, Faculty of Agriculture,

University of Tehran, Karaj, Iran

Full list of author information is available at the end of the article
}

(c) The Author(s). 2019 Open Access This article is distributed under the terms of the Creative Commons Attribution 4.0 International License (http://creativecommons.org/licenses/by/4.0/), which permits unrestricted use, distribution, and reproduction in any medium, provided you give appropriate credit to the original author(s) and the source, provide a link to the Creative Commons license, and indicate if changes were made. The Creative Commons Public Domain Dedication waiver (http://creativecommons.org/publicdomain/zero/1.0/) applies to the data made available in this article, unless otherwise stated. 


\section{Background}

Bread wheat (Triticum aestivum L.) is a staple crop both in developing and developed countries and there is a constant need to balance supply and demand [1]. The projected increase of human population is expected to increase the demand of wheat thereby highlighting the need for plant breeders to utilize all accessible tools to find new ways to sustainably increase the production of bread wheat over the coming decades $[1,2]$. Wheat production increased significantly after green revolution in the 1960s and 1970s by better farm management practices and introduction of well-adapted wheat varieties. However, the global consumption of wheat has also steadily been increasing due to population boom [1]. The comparison between wheat production and consumption in 1962 and 2012 shows a substantial increase in demand for wheat, with China, EU, India, and the USA being the major consumers. However, looking at the increase in demand over the 50-year period, it becomes clear that Asian countries, including Indonesia, Bangladesh, and Thailand are at the top of the list [1]. There are several factors drastically limiting wheat production throughout the world, such as water deficiency, salt and cold stress, resulting in significant losses in both grain and biomass [3, 4].

Understanding drought tolerance mechanisms and identifying loci responsible for mediating drought tolerance are key steps for any breeding approach aimed at increasing stress tolerance induced by water deficiency in bread wheat. Recent progress in sequencing technologies have become genome databases available for model plants, such as Arabidopsis thaliana [5], Oryza sativa [6], and a number of important crop species, including Hordeum vulgare [7] and T. aestivum [8]. The availability of large-scale genomic resources provides an opportunity to discover genetic and molecular mechanisms behind plant responses to different environmental stresses. As most agronomically important traits are likely controlled by a large number of genes, quantitative trait loci (QTL) mapping has been widely used to dissect the genetic architecture of such traits [9-11]. However, QTL-mapping has several drawbacks, such as low resolution and a limited number of alleles that can be screened per study. The high-throughput genotyping technologies providing large number of single nucleotide polymorphism (SNP) data has drastically improved the resolution of QTL mapping by providing highresolution linkage maps [12].

Furthermore, due to the increased availability of largescale genomic resources, genome-wide association studies (GWAS) are now a viable alternative to QTL mapping for dissecting the genetic architecture of quantitative traits [13]. In comparison to QTL-mapping, GWAS help accelerates the assessment of a more representative set of individuals in both time and cost-effective way [14]. GWAS are based on establishing correlations between genotype and phenotype, with the idea that linkage disequilibrium (LD) has been formed in a population across generations so that regions harboring QTLs can be detected even if the causal mutations aren't necessarily included among the set of available genetic markers. Access to highdensity genotyping spanning the entire genome makes GWAS invaluable tools for identifying genomic regions underlying the observed phenotypes. Several recent studies have successfully applied GWAS to identify the genetic basis of important traits in a number of crop species, including rice [15], barley [16], corn [17] and wheat [18]. These studies have also provided information about MTAs, which can help breeders in marker-assisted selection. In particular, a number of recent studies have focused on wheat by identifying QTLs associated with grain yield and related traits [19-21].

Genotyping by sequencing (GBS) can provide access to a large number of SNPs in a cost-efficient manner but it is often plagued by a high fraction of missing data that can limit the accuracy of any genome-wide association study. One approach to deal with missing data is through imputation and this has successfully been implemented in many studies on human and plant genomes [22-26]. Imputation can increase the number of variants that are included in a GWAS by relying on linkage information derived from common haplotypes after considering SNPs which are not directly genotyped [27]. Low depth sequencing and library complexity may contribute to missing information in SNP data and genotype imputation can thus be utilized to partly compensate for such issues through available reference genomes without the need for additional expensive resequencing $[28,29]$. The main objective of the current study was to perform a GWAS experiment using GBSSNP data [30] and SNPs imputed based on the W7984 reference genome for bread wheat, which has previously been demonstrated to yield the highest imputation accuracy [31]. A set of three categories of agronomic traits were measured among Iranian wheat landraces and varieties and employed in an association study to explore putative QTLs to identify genes, which may be involved in important developmental pathways providing drought tolerance. The second objective of the study was to determine if there are any differences in the results produced using the original SNP data compared to the imputed SNP data by assessing the influence of imputation on MTAs.

\section{Results}

\section{Phenotypic evaluation}

The datasets for well-watered and rain-fed conditions were analyzed separately. ANOVA identified significant differences $(P<0.01)$ among varieties and landraces for all studied traits under both environments for two years, except thousand kernel weight under rain-fed conditions 
(Additional file 2: Tables S2 and 3). Under rain-fed conditions, early emergence was delayed, yet genotypes completed their lifespan earlier compared to the wellwatered conditions through a 14.9 days reduction in physiological maturity (Table 1 ). The grain filling period was 27.2 and 24.0 days for well-watered and rain-fed conditions, respectively. The greatest variation under well-watered conditions was observed for seed number per spike and thousand kernel weight (std. deviation 7.35 and 7.10, respectively), whereas plant height and peduncle length were more variable under rain-fed conditions (SD 15.55 and 7.26, respectively). A significant positive association was observed between grain yield, spike weight, seed number, thousand kernel weight, leaf greenness, and grain filling period under well-watered conditions $(P<0.01)$, whereas phenology traits and canopy temperature were negatively correlated with grain yield $(P<0.01$ and 0.05 , Additional file 2: Table S4). Under rain-fed conditions, grain yield was negatively correlated with phenological traits, plant height, peduncle length, spike length, and canopy temperature, whereas significant positive correlations were observed between grain yield, spike weight, seed number per spike, and thousand kernel weight $(P<0.01$, Additional file 2: Table S5).

\section{Evaluation of SNP markers}

A total of $458,363,607$ unique reads were identified in total 566,439,207 reads after sequencing ( 81\% nonredundant reads). After de-duplication and alignment, 133,039 SNPs were called for which 10,938 had <10\% missing data, heterozygosity $<10 \%$ and a minor allele frequency (MAF) $>1 \%$. These SNPs were selected for further analysis. Among the 10,938 SNPs identified, the highest $(2835,25.92 \%)$ and lowest $(597,5.46 \%)$ number of markers were observed for MAFs in the range of $0.01-0.1$, and $0.45-0.50$, respectively (Fig. 1). In addition, we obtained a set of 46,862 imputed SNPs using the W7984 reference genome and these SNPs were also used to estimate genetic diversity.

\section{Linkage disequilibrium (LD)}

The analysis of linkage disequilibrium shows that LD differs between sub-genomes, chromosomes, and across each chromosome and that LD generally declines with increasing distance between SNPs. A total of 368,310 marker pairs (MP, according to combinations of SNPs across different chromosomes) with average squared allele frequency correlations or $r^{2}=0.132$ were observed in varieties, of which 96,541 (26.2\%) had significant linkage at $P<0.01$ (Table 2). Around $93 \%$ of all MPs and $94 \%$ of significant MPs were located at distances $<10$ cM. Genomes B and D harbored the highest and lowest number of MPs (182,271, 49.49\% and 50,395, 13.68\%), respectively. Moreover, the strongest LD was observed between MPs on chromosome 2D (0.296), followed by chromosome 1D (0.214).

Performing a similar analysis on landraces identified a total of 405,738 MPs with an average LD of 0.097 which is considerably lower than in varieties (Table 2). However, a greater fraction of significant MPs $(29.31 \%)$ was observed in the landrace data. Eighty-nine percent and

Table 1 Descriptive statistics for agronomic traits of Iranian wheat accessions under well-watered and rain-fed conditions

\begin{tabular}{|c|c|c|c|c|c|c|}
\hline \multirow[b]{2}{*}{ Trait } & \multicolumn{3}{|l|}{ well-watered } & \multirow[b]{2}{*}{ Range } & \multicolumn{2}{|c|}{ Rain-fed } \\
\hline & Range & Mean & Std. Deviation & & Mean & Std. Deviation \\
\hline$\overline{\mathrm{DE}}$ & $19.9-27.5$ & 24.7 & 1.6 & $22.9-37.8$ & 28.5 & 2.4 \\
\hline $\mathrm{DH}$ & $160.0-188$ & 175.9 & 9 & $135.4-179.8$ & 167.1 & 6.6 \\
\hline DA & 167.6-196.6 & 184.9 & 5.9 & $158.7-185.9$ & 173 & 6.1 \\
\hline DM & $194.6-224.7$ & 211.9 & 6.2 & $183.2-209.4$ & 197 & 6.4 \\
\hline GFP & $20.5-35.5$ & 27.2 & 2.5 & $16.7-31.5$ & 24 & 2.8 \\
\hline $\mathrm{H}$ & - & - & - & $53-130$ & 87.1 & 15.6 \\
\hline$P L$ & - & - & - & $19.9-64.2$ & 36.3 & 7.3 \\
\hline SW & $1.1-4$ & 2.5 & 0.44 & $0.90-2.6$ & 1.7 & 0.35 \\
\hline SL & $5.7-18.7$ & 10.8 & 2.1 & $6.7-13.2$ & 9.9 & 1.1 \\
\hline GY & $0.66-2.8$ & 1.8 & 0.35 & $0.47-1.8$ & 1.1 & 0.24 \\
\hline SN & $23.8-72.3$ & 43.4 & 7.4 & $23.5-57.9$ & 37.7 & 7 \\
\hline TKW & $14.9-74.5$ & 41.0 & 7.1 & $15.6-44.7$ & 29.6 & 5.7 \\
\hline LG & $37.94-66.1$ & 51.3 & 4.9 & $33.7-62.5$ & 49.1 & 5.1 \\
\hline$C T$ & $20.1-27.8$ & 23.9 & 1.4 & $22.1-33.1$ & 27.6 & 1.6 \\
\hline
\end{tabular}

DE: Days to emergence, DH: Days to heading, DA: Days to anthesis, DM: Days to physiological maturity, GF: Grain filling period, H: Plant height (cm), PL: Peduncle length $(\mathrm{cm})$, SW: Spike weight $(\mathrm{g}), \mathrm{SL}$ : Spike length $(\mathrm{cm}), \mathrm{GY}$ : Grain yield (g per plant), SN: Seed number per spike, TKW: Thousand kernel weight (g), LG: Leaf greenness, $\mathrm{CT}$ : Canopy temperature $\left({ }^{\circ} \mathrm{C}\right)$ 


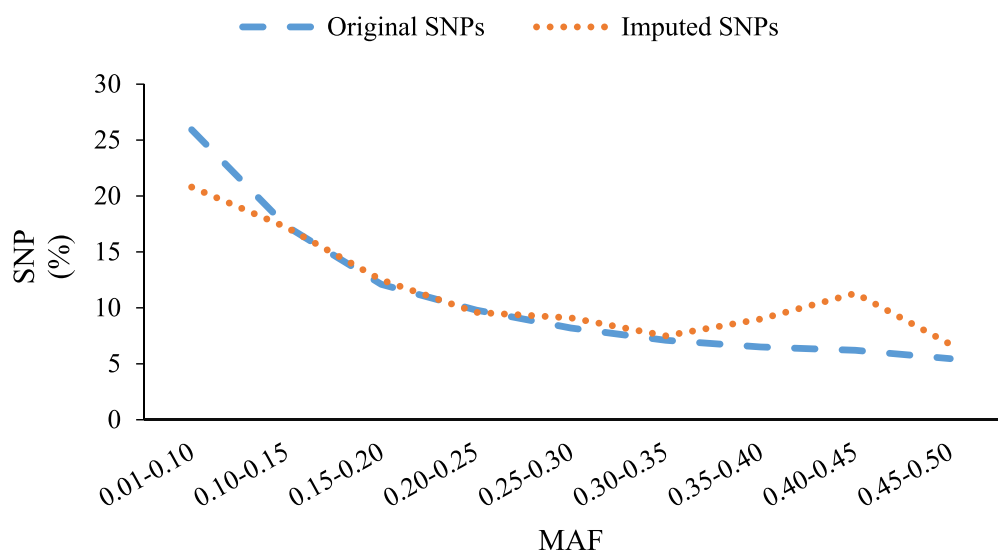

Fig. 1 The distribution of SNPs according to different MAF for the original and imputed datasets

Table 2 A summary of observed LD among marker pairs and the number of significant marker pairs per chromosome and genome using original SNPS

\begin{tabular}{|c|c|c|c|c|c|c|c|c|}
\hline \multirow[t]{2}{*}{ Chromosome } & \multicolumn{4}{|l|}{ varieties } & \multicolumn{4}{|c|}{ Landraces } \\
\hline & TNMP & $r^{2}$ & $\begin{array}{l}\text { Distance } \\
\text { (cM) }\end{array}$ & SMP & TNMP & $r^{2}$ & $\begin{array}{l}\text { Distance } \\
\text { (cM) }\end{array}$ & SMP \\
\hline $1 \mathrm{~A}$ & 17,718 & 0.1106 & 5.9776 & $4338(24.48)$ & 22,647 & 0.0813 & 5.1594 & 5835 (25.77) \\
\hline 1B & 24,887 & 0.1465 & 3.8458 & 6559 (26.36) & 28,687 & 0.0963 & 3.8538 & 9506 (33.14) \\
\hline 1D & 10,568 & 0.2104 & 9.3147 & 3773 (35.70) & 13,133 & 0.1195 & 10.5104 & 4337 (33.02) \\
\hline $2 \mathrm{~A}$ & 21,453 & 0.1341 & 4.2847 & 5487 (25.58) & 25,306 & 0.1154 & 3.9947 & 8527 (33.70) \\
\hline $2 B$ & 36,066 & 0.1327 & 3.3614 & $9728(26.97)$ & 33,314 & 0.0925 & 3.4718 & 10,666 (32.02) \\
\hline $2 \mathrm{D}$ & 12,523 & 0.2959 & 6.0466 & 4741 (37.86) & 16,319 & 0.1976 & 6.1044 & 5473 (33.54) \\
\hline $3 \mathrm{~A}$ & 21,696 & 0.1159 & 7.5667 & 4831 (22.27) & 19,424 & 0.0748 & 7.4690 & $4629(23.83)$ \\
\hline 3B & 31,120 & 0.1327 & 3.8233 & $8632(27.74)$ & 33,719 & 0.0974 & 3.8089 & $10,860(32.21)$ \\
\hline $3 D$ & 4274 & 0.1117 & 14.9170 & 713 (16.68) & 7601 & 0.0994 & 17.2124 & $1782(23.44)$ \\
\hline $4 \mathrm{~A}$ & 16,982 & 0.1484 & 6.6126 & 4548 (26.78) & 17,092 & 0.1164 & 7.0726 & $5002(29.27)$ \\
\hline $4 \mathrm{~B}$ & 11,382 & 0.1679 & 6.8900 & 3505 (30.79) & 8498 & 0.0608 & 8.6884 & $1554(18.29)$ \\
\hline 4D & 1918 & 0.1836 & 22.8230 & $492(25.65)$ & 2329 & 0.1422 & 22.8137 & 1037 (44.53) \\
\hline $5 \mathrm{~A}$ & 15,226 & 0.1217 & 6.3518 & 3614 (23.74) & 17,683 & 0.0867 & 6.8862 & $5281(29.86)$ \\
\hline $5 B$ & 28,463 & 0.1427 & 5.4429 & $8533(29.98)$ & 29,599 & 0.0728 & 5.4563 & 7454 (25.18) \\
\hline $5 \mathrm{D}$ & 5524 & 0.1049 & 23.4950 & 848 (15.35) & 6152 & 0.0742 & 27.2205 & $1339(21.77)$ \\
\hline $6 \mathrm{~A}$ & 16,916 & 0.1120 & 6.4506 & 3578 (21.15) & 18,115 & 0.1161 & 6.4866 & $6739(37.20)$ \\
\hline $6 \mathrm{~B}$ & 23,696 & 0.1456 & 3.5509 & $7080(29.88)$ & 28,304 & 0.0729 & 3.9161 & 7225 (25.53) \\
\hline $6 \mathrm{D}$ & 6899 & 0.1150 & 16.5648 & 1375 (19.93) & 8454 & 0.0828 & 16.0911 & $2112(24.98)$ \\
\hline $7 \mathrm{~A}$ & 25,653 & 0.1506 & 4.8667 & $6132(23.90)$ & 30,419 & 0.1052 & 4.7313 & 8988 (29.55) \\
\hline 7B & 26,657 & 0.1136 & 4.1457 & $5843(21.92)$ & 27,880 & 0.0807 & 3.9193 & 7821 (28.05) \\
\hline 7D & 8689 & 0.1822 & 16.5774 & 2191 (25.22) & 11,063 & 0.0996 & 15.7344 & $2766(25.00)$ \\
\hline A genome & 135,644 & 0.1289 & 5.9344 & 32,528 (23.98) & 150,686 & 0.0994 & 5.9714 & $45,001(29.86)$ \\
\hline B genome & 182,271 & 0.1372 & 4.1911 & $49,880(27.37)$ & 190,001 & 0.0819 & 4.7307 & 55,086 (28.99) \\
\hline D genome & 50,395 & 0.1928 & 13.2910 & 14,133 (28.04) & 65,051 & 0.1165 & 16.5267 & 18,846 (28.97) \\
\hline Total & 368,310 & 0.1321 & 6.0783 & $96,541(26.21)$ & 405,738 & 0.0968 & 6.3498 & $118,933(29.31)$ \\
\hline
\end{tabular}

TNMP: Total no. of marker pairs, SMP: Significant marker pairs $(P<0.01)$ 
$88 \%$ of the total and significant MPs had distances $<10$ cM. Moreover, the greatest number of MPs was observed in the B genome $(190,001)$. The overall number of SNPs located in different sub-genomes in landraces was slightly higher than in varieties. Similarly, LD was highest in chromosome 2D (0.198). The LD decay is visualized in Additional file 3: Figs. S2-4. LD on chromosomes of the D genome shows a distinct trend, where LD decay occurred more slowly compared to either A or $\mathrm{B}$ genomes. For most chromosomes of the $\mathrm{A}$ and $\mathrm{B}$ genomes, LD declined to 0.1 over distances of $<5 \mathrm{cM}$, whereas the corresponding distances were $5-10 \mathrm{cM}$ in D-genome.

\section{Population structure and kinship matrix}

We evaluated population structure using the variancecovariance matrix of individuals (Kinship matrix) obtained from both original and imputed SNPs. For both datasets, the analyses identified three main groups with varying degrees of admixture. For the original dataset, the first two principal components explained $17.2 \%$ of the genetic variance (Fig. 2a), whereas the variance was $23.2 \%$ for the imputed SNPs (Fig. 2b). Moreover, analysis of population structure showed the highest value of $\Delta \mathrm{K}$ for $K=3$.

Group I contains 69 accessions with 66 varieties and 3 landraces; Group II contains 120 accessions with 102 landraces and 18 varieties, and Group III contains 103 landraces and 6 varieties (Fig. 3a). Accessions also clustered into three main groups when we used the imputed SNPs, where Group I contains 113 accessions with 108 landraces and 5 varieties, Group II contains 74 studies with 70 varieties and 4 landraces; Group III contains 110 accessions with 97 landraces and 13 varieties (Fig. 3b). According to the original SNP data, twenty-four varieties appear to be admixed with the two landrace groups, while for the imputed SNP data, only 19 such admixed varieties were identified. The admixed varieties originated from Iranian landraces and varieties including Shahi, 4820, Mahdavi, Azadi, Ghods, Neishabour and Sivand derived from other materials.

A neighbor-joining tree of all varieties also clearly showed the clustering into three subgroups for both datasets (Fig. 4), with the exception of the varieties Khazar1, Akova, Frontana, and Alborz which shifted into two series of 12 and 25 varieties of two neighbor's groups (Fig. 4b). Even though landraces clustered into three groups based on the two SNPs datasets, their differentiation was more clearly distinguished using imputed SNPs (Fig. 5). Accessions PI627236 and PI625433 were grouped into the same group using original SNPs, while accession PI625433 shifted into the largest group of landraces and the distribution of the two small groups changed when the imputed SNPs were used for clustering.

\section{MTAs for agronomic and physiological traits}

A total of 313 MTAs were identified using the original SNP dataset at a significance value of $-\log _{10} P>3$ for both well-watered and rain-fed conditions. A total of 394 MTAs were detected for the imputed data (Table 3). The highest number of MTAs was located on chromosomes from the B genome for both the original and imputed SNPs whereas D genome showed the smallest number of MTAs. Among the traits that were studied
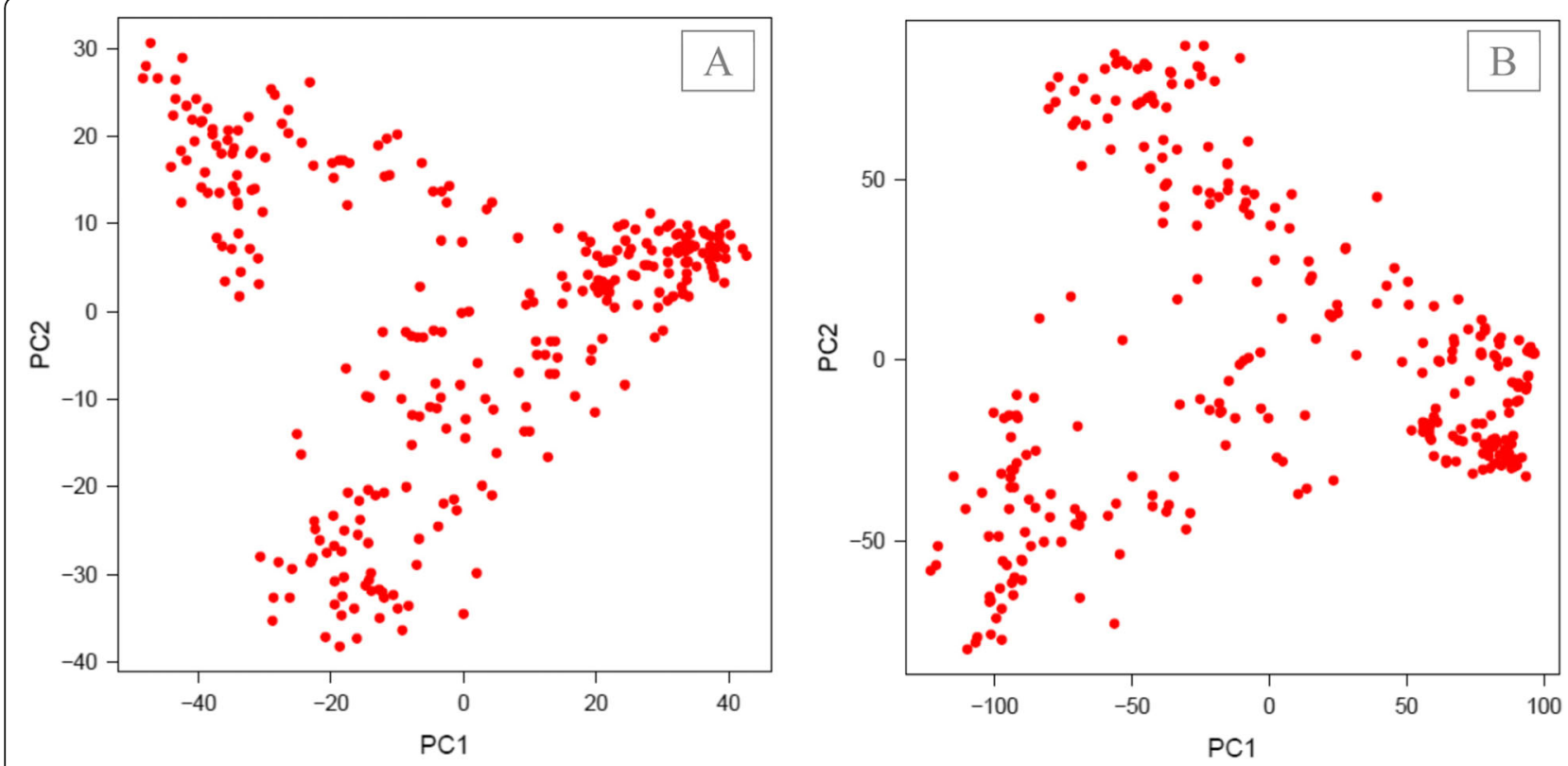

Fig. 2 Principal component analysis of Iranian accessions using original SNPS (A), and imputed SNPS (B) 

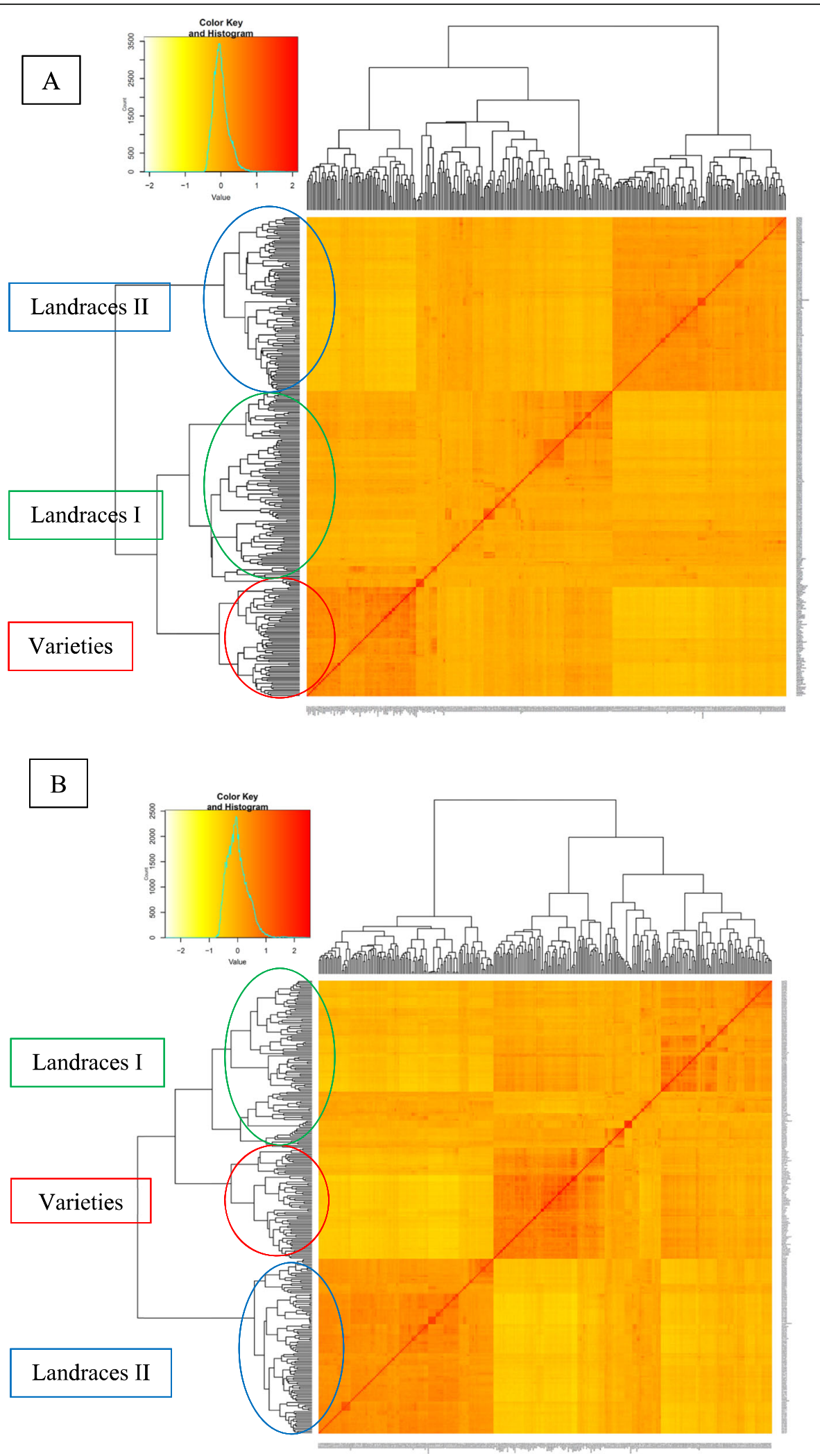

Fig. 3 Cluster analysis using Kinship matrix of original data (A) and imputed data (B) for Iranian wheat accessions 

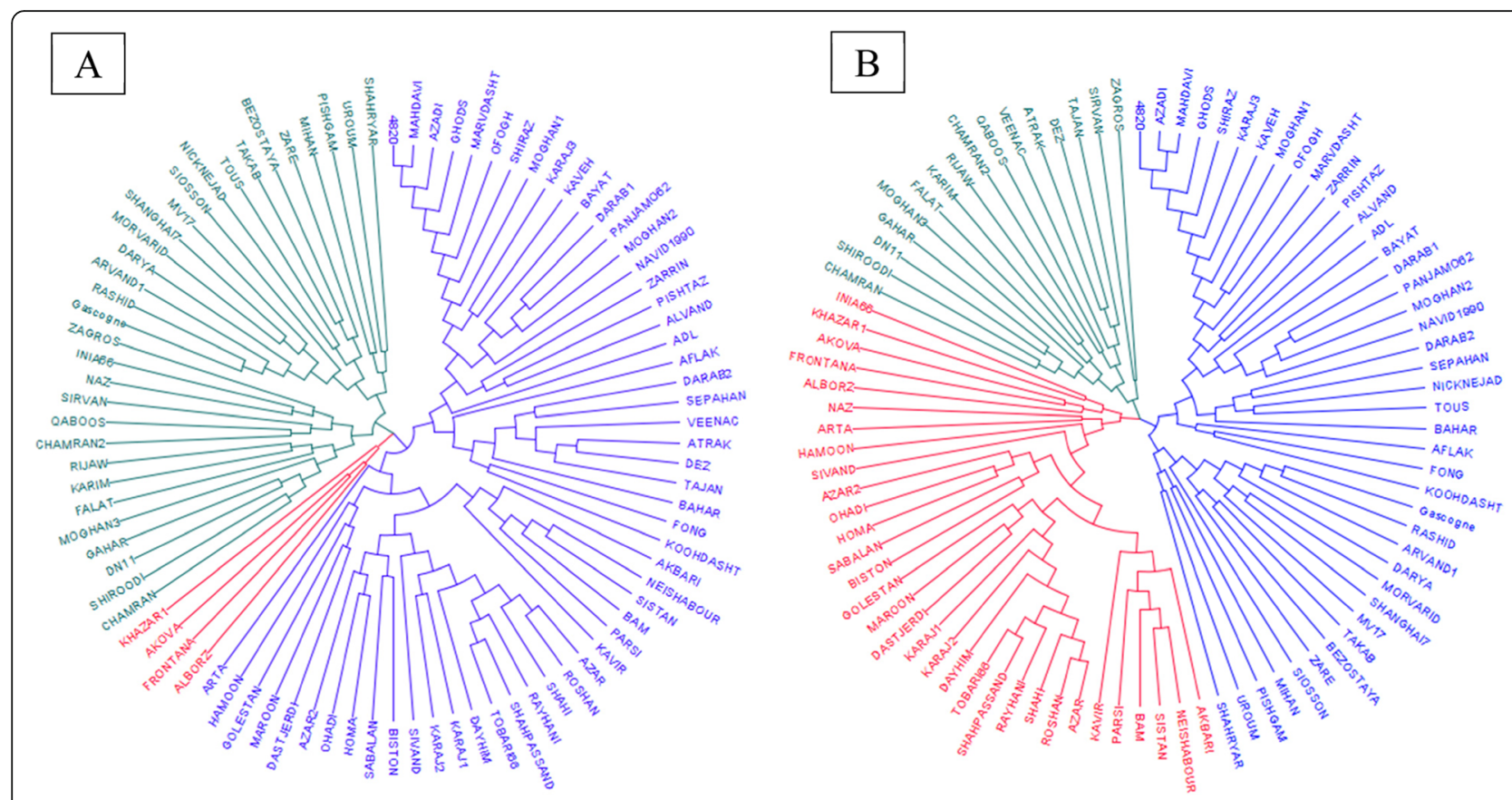

Fig. 4 The dendrogram of Neighbor-Joining clustering constructed using 10,938 (A) and 46,862 (B) SNPs and 90 Iranian hexaploid wheat varieties

under well-watered conditions, grain filling period and spike length showed the highest number of associated MTAs for both original and imputed SNPs, respectively. Moreover, 13 and 4 MTAs were observed for grain yield per plant under well-watered conditions using original and imputed datasets, respectively. For drought stress conditions, 23 MTAs were observed for grain filling period and peduncle length based on non-imputed SNPs, whereas 10 and 18 MTAs showed significant association with these traits using imputed SNPs. However, the highest number of imputed MTAs was obtained for seed number per spike (25 MTAs).
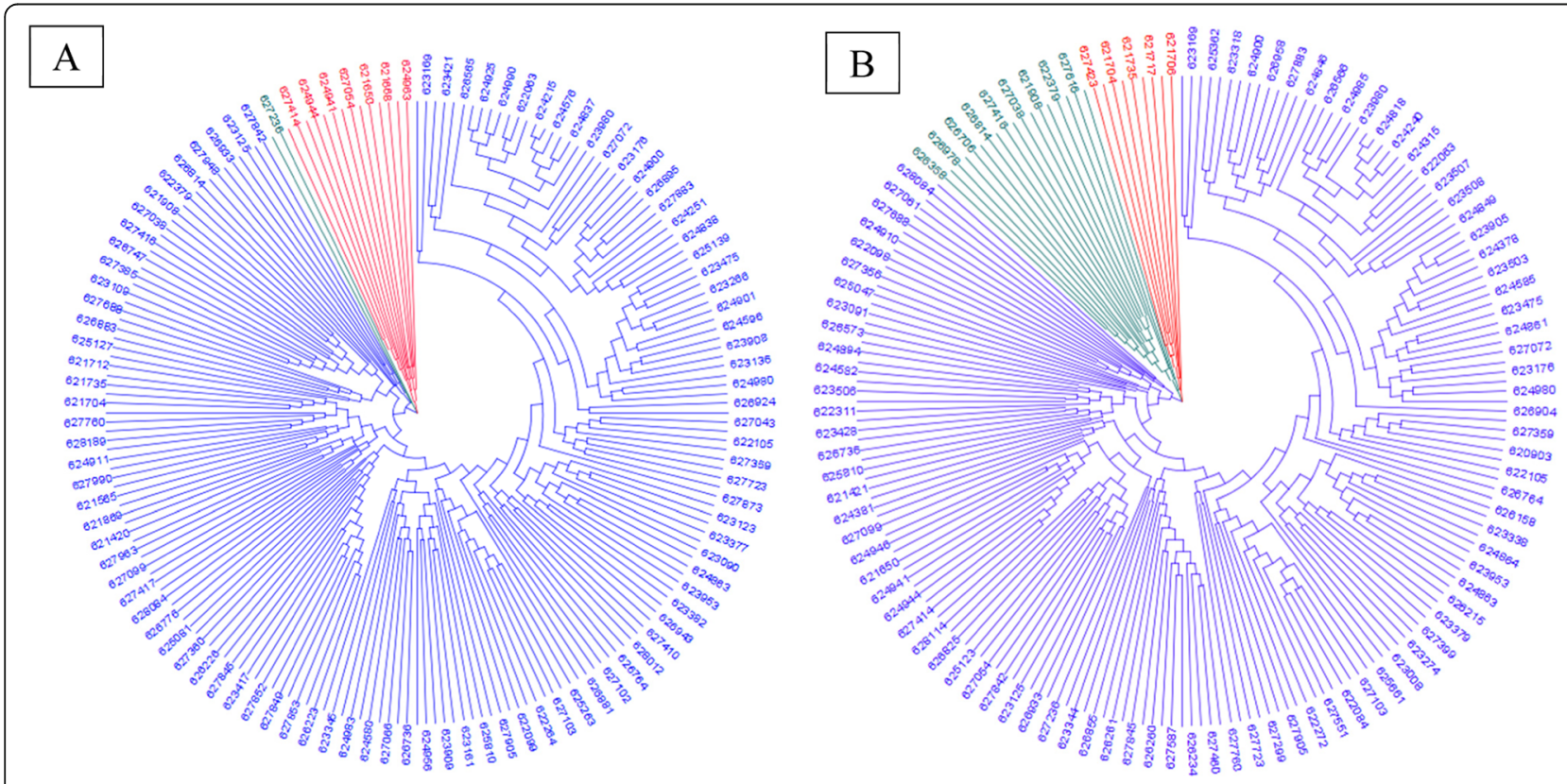

Fig. 5 The dendrogram of Neighbor-Joining clustering constructed using 10,938 (A) and 46,862 (B) SNPs and 208 Iranian hexaploid wheat landraces collected from different zones 
Table 3 A summary of marker-trait associations for agronomic traits of Iranian wheat accessions under well-watered and rain-fed conditions

\begin{tabular}{|c|c|c|c|c|}
\hline \multirow[t]{2}{*}{ Genome } & \multicolumn{2}{|l|}{ Well-watered } & \multicolumn{2}{|l|}{ Rain-fed } \\
\hline & Original data & Imputed data & Original data & Imputed data \\
\hline MTA & 117 & 177 & 196 & 217 \\
\hline Genome A & 36 & 89 & 60 & 85 \\
\hline Genome B & 51 & 83 & 92 & 110 \\
\hline Genome D & 12 & 5 & 28 & 22 \\
\hline Unassembled Chromosomes & 18 & - & 16 & - \\
\hline
\end{tabular}

For the original SNP data, the highest number of significant markers under well-watered conditions was observed on chromosomes $2 \mathrm{~B}$ and $7 \mathrm{~B}$ with 12 and 11 SNPs respectively, followed by chromosomes $2 \mathrm{~A}, 3 \mathrm{~A}$, and $6 \mathrm{~B}$ with 8 markers per chromosome. Under rain-fed conditions, a total of 29 and 15 associated SNPs were identified on chromosome $5 \mathrm{~B}$ and 6B. For the imputed dataset, 47, 32, and 26 significant markers were identified on chromosomes 4B, 1A, and 5B under wellwatered conditions, whereas 85,40 , and 28 markers were identified on chromosomes $5 \mathrm{~B}, 4 \mathrm{~A}$, and $1 \mathrm{~A}$ while using the imputed-SNPs under rain-fed conditions. A number of markers on chromosomes A and B showed pleiotropic effects among different traits.

\section{Gene annotation}

The gene ontology of the 313 MTAs that we identified using the original SNP dataset shows that 27 and 27.5\% of the MTAs were located within protein-coding genes under well-watered and rain-fed conditions, respectively (Additional file 4: Tables S6, and 7). In contrast, among 394 MTAs identified using the imputed SNP data set, 22.6 and $16.6 \%$ were located within coding genes under well-watered and rain-fed conditions, respectively (Additional file 4: Tables S8, and 9). The genes with MTAs mostly encode proteins involved in ubiquitination, oxidation-reduction, protein phosphorylation, histone deubiquitination, negative regulation of transcription, response to abscisic acid, catabolic process, multicellular organism development, xanthophyll biosynthetic, response to UV, ion transportation, cytokinin biosynthetic, DNA methylation, DNA replication, cellular response to DNA damage stimulus, response to oxidative stress, cellular protein modification process, and carbohydrate metabolic process.

We have summarized the results of the SNPs showing the strongest association in Tables 4 to 7 . Under wellwatered conditions, 5 and 7 markers within coding genes were located on chromosomes $1 \mathrm{~A}, 4 \mathrm{~B}, 4 \mathrm{D}, 5 \mathrm{~A}, 5 \mathrm{~B}, 6 \mathrm{~B}$ and $7 \mathrm{~B}$ for original and imputed SNPs, respectively (Tables 4 and 5). These markers were associated with canopy temperature, seed number per spike, thousand kernel weight, grain filling period, grain yield, spike weight and length, leaf greenness and days to emergence. Most of these SNPs are located on the B genome, followed by the A genome and finally the D genome (Tables 4 and 5). Under rain-fed conditions, 14 and 11 SNPs were associated with genes involved in regulating spike weight and length, thousand kernel weight, plant height, peduncle length, leaf greenness, grain filling period, seed number, grain yield, canopy temperature, days to emergence, heading and physiological maturity (Tables 6 and 7). All these markers were located on the $\mathrm{B}$ and $\mathrm{A}$ genomes and there was no significantly associated marker located on the $\mathrm{D}$ genome under rain-fed conditions. Overall, the B genome contains a considerable portion of all highly significant SNPs for agronomic traits. Markers rs36032 on chromosome 1A and rs56337 on chromosome 7A were linked to genes that are involved in providing grain yield under well-watered and rain-fed environments, respectively.

\section{Mining of highly associated favorable alleles}

In the current study, SNPs with positive effects, causing an increase in grain yield, seed number, thousand kernel weight, grain filling period, spike weight, leaf greenness and the reduction in the days to emergence, days to heading, days to anthesis, days to physiological maturity, canopy temperature, plant height, peduncle length and spike length were defined as favorable alleles. The phenotypic effect of strongly associated SNPs under well-watered conditions using both original and imputed dataset was quantified using $a i$, where a reduction was observed for rs62576 by 0.72 in canopy temperature, rs34314 by 1.09 , rs 40819 by 0.70, rs 36808 by 0.50 in spike length, and rs 57386 by 0.11 in days to emergence (Table 8). The positive increasing effect of rs48893 and rs10316 on seed number per spike was 0.96 and 1.20 , respectively. Moreover, thousand kernel weight, grain filling period, grain yield and leaf greenness were increased by $0.77 \mathrm{~g}, 0.90 \mathrm{~d}, 0.01 \mathrm{~g}$, and 1.82 , respectively. Spike weight was associated with two markers, rs36032, and rs736, which increased the trait by $0.02 \mathrm{~g}$ and $0.33 \mathrm{~g}$, respectively. The phenotypic variance explained (PVE) by the associated SNPs ranged from 7 to $40 \%$ (Table 8). 
Table 4 Description of expected MTAs using original SNPs for agronomic traits of Iranian wheat accessions exposed to the wellwatered conditions

\begin{tabular}{|c|c|c|c|c|c|c|c|}
\hline Row & Marker & sequence & Trait & Chromosome & $\begin{array}{l}\text { Position } \\
\text { (bp) }\end{array}$ & Molecular process & $\begin{array}{l}\text { Biological } \\
\text { process }\end{array}$ \\
\hline 1 & rs62576 & $\begin{array}{l}\text { TGCAGTTACGGATGGCAGTCATCTGGTCCATGAA } \\
\text { TCATGACAGAGGCACCTGCTCCATAAACAG_47 }\end{array}$ & $\begin{array}{l}\text { Canopy } \\
\text { temperature }\end{array}$ & $1 \mathrm{~A}$ & $\begin{array}{l}570,131 \\
664-570 \\
140,605\end{array}$ & oxidoreductase activity, & $\begin{array}{l}\text { oxidation- } \\
\text { reduction } \\
\text { process }\end{array}$ \\
\hline 2 & rs48893 & $\begin{array}{l}\text { TGCAGGCTCCGCTAAACCCTAGACTTGACGGCGA } \\
\text { GGGTGCGTCGGGTGGGGAAAGGGGGAGAAA_11 }\end{array}$ & $\begin{array}{l}\text { Seed } \\
\text { number }\end{array}$ & $4 \mathrm{D}$ & $\begin{array}{l}318,493 \\
437-318 \\
496,592\end{array}$ & GTPase activity & - \\
\hline 3 & rs4772 & $\begin{array}{l}\text { TGCAGACTCACACACAAGCTGCTACAACTA } \\
\text { AGCGCTGGGCAGATACATCCACCCGAGA } \\
\text { TCGGAA_44 }\end{array}$ & $\begin{array}{l}\text { Thousand } \\
\text { kernel } \\
\text { weight }\end{array}$ & $5 B$ & $\begin{array}{l}435,156 \\
029-435 \\
159,077\end{array}$ & $\begin{array}{l}\text { protein kinase activity } \\
\text { ATP binding }\end{array}$ & $\begin{array}{l}\text { protein } \\
\text { phosphorylation }\end{array}$ \\
\hline 4 & rs46504 & $\begin{array}{l}\text { TGCAGGCATATGCTCGCCCCACATGTTCGTAGAC } \\
\text { AGGCTATCCTGCCGTTACGCATTGTGGTAC_30 }\end{array}$ & $\begin{array}{l}\text { Grain filling } \\
\text { period }\end{array}$ & $6 B$ & $\begin{array}{l}534,921 \\
073-534 \\
927,092\end{array}$ & $\begin{array}{l}\text { guanyl-nucleotide } \\
\text { exchange factor } \\
\text { activity } \\
\text { Rho guanyl-nucleotide } \\
\text { exchange factor } \\
\text { activity }\end{array}$ & - \\
\hline 5 & rs10316 & $\begin{array}{l}\text { TGCAGATTGGGCTTGAGGAAATCTAACAAAACTT } \\
\text { GGTGGATCGGCAAAGCCTGGATGAAATTCA_6 }\end{array}$ & $\begin{array}{l}\text { Seed } \\
\text { number }\end{array}$ & $7 B$ & $\begin{array}{l}675,187 \\
632-675 \\
190,824\end{array}$ & DNA binding & - \\
\hline
\end{tabular}

Table 5 Description of expected MTAs using imputed SNPs for agronomic traits of Iranian wheat accessions exposed to the wellwatered conditions

\begin{tabular}{|c|c|c|c|c|c|c|c|}
\hline Row & Marker & sequence & Trait & Chromosome & $\begin{array}{l}\text { Position } \\
\text { (bp) }\end{array}$ & Molecular process & Biological process \\
\hline 1 & rs36032 & $\begin{array}{l}\text { TGCAGCTCATCACTAGTCTCGCGCTC } \\
\text { GGGCAGCAGGACCGAGCTCGTCTC } \\
\text { GCGCCCG_25 }\end{array}$ & $\begin{array}{l}\text { Grain yield } \\
\text { and spike } \\
\text { weight }\end{array}$ & $1 \mathrm{~A}$ & $\begin{array}{l}\text { 206,792, } \\
054-206 \\
805,538\end{array}$ & $\begin{array}{l}\text { nucleotide binding } \\
\text { DNA binding } \\
\text { damaged DNA binding } \\
\text { ATP binding } \\
\text { mismatched DNA } \\
\text { binding }\end{array}$ & $\begin{array}{l}\text { DNA repair } \\
\text { pyrimidine dimer repair } \\
\text { cellular response to DNA } \\
\text { damage stimulus } \\
\text { negative regulation of } \\
\text { reciprocal meiotic } \\
\text { recombination }\end{array}$ \\
\hline 2 & rs34075 & $\begin{array}{l}\text { TGCAGCGTTCGACCAGCTCATCACCC } \\
\text { GCTTCCGAGATCGGAAGAGCGGGA } \\
\text { TCACCGACTGCCCA_19 }\end{array}$ & $\begin{array}{l}\text { Leaf } \\
\text { greenness }\end{array}$ & $1 \mathrm{~A}$ & $\begin{array}{l}60,954 \\
701-60 \\
956,424\end{array}$ & $\begin{array}{l}\text { peroxidase activity } \\
\text { oxidoreductase activity } \\
\text { heme bindingmetal ion } \\
\text { binding }\end{array}$ & $\begin{array}{l}\text { response to oxidative } \\
\text { stresshydrogen peroxide } \\
\text { catabolic process } \\
\text { oxidation-reduction } \\
\text { process } \\
\text { cellular oxidant } \\
\text { detoxification }\end{array}$ \\
\hline 3 & rs34314 & $\begin{array}{l}\text { TGCAGCTAACTAGCCTGAGATAATGC } \\
\text { CAGCAACTCTGCTCGGTAGCTTTC } \\
\text { TTAAGAAGGCCTTA_45 }\end{array}$ & $\begin{array}{l}\text { Spike } \\
\text { length }\end{array}$ & $4 B$ & $\begin{array}{l}386,744 \\
409-386 \\
747,753\end{array}$ & $\begin{array}{l}\text { catalytic activity tRNA- } \\
\text { specific adenosine de- } \\
\text { aminase activity } \\
\text { zinc ion binding } \\
\text { hydrolase activity }\end{array}$ & tRNA modification \\
\hline 4 & rs736 & $\begin{array}{l}\text { TGCAGAAAGGTACCACTCATTCGTAC } \\
\text { ATCACTCCAACTGATGTATGAAGGTTGT } \\
\text { TCATGGCGAC_18 }\end{array}$ & $\begin{array}{l}\text { Spike } \\
\text { weight }\end{array}$ & $4 \mathrm{~B}$ & $\begin{array}{l}481,233 \\
765-481 \\
237,258\end{array}$ & hydrolase activity & $\begin{array}{l}\text { phosphatidylinositol } \\
\text { dephosphorylation }\end{array}$ \\
\hline 5 & rs40819 & $\begin{array}{l}\text { TGCAGCTTCCATTTCATTCCTTCCTG } \\
\text { CGCCATGGGTAACAAAAATTCAACTTCT } \\
\text { TCAGTAACA_32 }\end{array}$ & $\begin{array}{l}\text { Spike } \\
\text { length }\end{array}$ & $4 \mathrm{~B}$ & $\begin{array}{l}667,563 \\
369-667 \\
564,460\end{array}$ & protein binding & - \\
\hline 6 & rs57386 & $\begin{array}{l}\text { TGCAGTATCGCAAGAGTAAAATGAAG } \\
\text { TAGACAAAAACCTTGTATCATTAAAAGA } \\
\text { GGCAGTCACC_18 }\end{array}$ & $\begin{array}{l}\text { Days to } \\
\text { emergence }\end{array}$ & $5 \mathrm{~A}$ & $\begin{array}{l}467,397 \\
067-467 \\
403,109\end{array}$ & $\begin{array}{l}\text { serine-type } \\
\text { endopeptidase activity } \\
\text { serine-type peptidase } \\
\text { activity } \\
\text { serine-type } \\
\text { exopeptidase activity }\end{array}$ & proteolysis \\
\hline 7 & rs36808 & $\begin{array}{l}\text { TGCAGCTCCGTGTCAGTGGTGTCGCG } \\
\text { GGTGAGGCTCTTCTGCTCATCGGC } \\
\text { GCGGATCGGAACTT_44 }\end{array}$ & $\begin{array}{l}\text { Spike } \\
\text { length }\end{array}$ & $5 B$ & $\begin{array}{l}287,752 \\
969-287 \\
780,293\end{array}$ & $\begin{array}{l}\text { ATP binding } \\
\text { ATPase activity }\end{array}$ & - \\
\hline
\end{tabular}


Table 6 Description of expected MTAs using original SNPs for agronomic traits of Iranian wheat accessions exposed to the rain-fed conditions

\begin{tabular}{|c|c|c|c|c|c|c|c|}
\hline Row & Marker & sequence & Trait & Chromosome & $\begin{array}{l}\text { Position } \\
\text { (bp) }\end{array}$ & Molecular process & Biological process \\
\hline 1 & rs63808 & $\begin{array}{l}\text { TGCAGTTGAAGTCGCGGTGGATGACG } \\
\text { GCGGGGGAGGTGTGCTCGTGCAGAAACT } \\
\text { CCAGCGCGCG_49 }\end{array}$ & $\begin{array}{l}\text { Spike } \\
\text { weight }\end{array}$ & $1 \mathrm{~B}$ & $\begin{array}{l}457,750 \\
965-457 \\
756,510\end{array}$ & $\begin{array}{l}\text { protein kinase activity } \\
\text { ATP binding }\end{array}$ & $\begin{array}{l}\text { protein } \\
\text { phosphorylation }\end{array}$ \\
\hline 2 & rs2237 & $\begin{array}{l}\text { TGCAGAAGGGGACGCCTCGGAATCTA } \\
\text { CGGCAGAGGACCGCCTCAGCGGCCTTCC } \\
\text { CGACGGCGTC_30 }\end{array}$ & $\begin{array}{l}\text { Spike } \\
\text { length }\end{array}$ & $1 \mathrm{~B}$ & $\begin{array}{l}26,855 \\
662-26 \\
857,170\end{array}$ & $\begin{array}{l}\text { protein binding } \\
\text { (F-box domain) }\end{array}$ & - \\
\hline 3 & rs 26577 & $\begin{array}{l}\text { TGCAGCCTCCAATCGTGTACACACCTCCGT } \\
\text { AAACAGATCTCGATTCTTCACTCCCTGT } \\
\text { AGAGAG_5 }\end{array}$ & $\begin{array}{l}\text { Thousand } \\
\text { kernel } \\
\text { weight }\end{array}$ & $2 B$ & $\begin{array}{l}134,240 \\
300-134 \\
249,722\end{array}$ & $\begin{array}{l}\text { protein binding } \\
\text { (Armadillo) } \\
\text { Involved in } \\
\text { membrane }\end{array}$ & - \\
\hline 4 & rs15903 & $\begin{array}{l}\text { TGCAGCAGAGAATAATAGATGGAGGG } \\
\text { AGGGGTGGTGCAAGTATAGCACCCGAGA } \\
\text { TCGGAAGAGC_41 }\end{array}$ & $\begin{array}{l}\text { Spike } \\
\text { weight }\end{array}$ & $2 B$ & $\begin{array}{l}47,175 \\
539-47 \\
181,332\end{array}$ & $\begin{array}{l}\text { ADP binding } \\
\text { (NB-ARC) }\end{array}$ & - \\
\hline 5 & rs46075 & $\begin{array}{l}\text { TGCAGGCACGACCGCATGACCTTCTCGAAC } \\
\text { TTGGCGTCCTGGCATGGGCGAGCGCAG } \\
\text { ACTCGA_25 }\end{array}$ & $\begin{array}{l}\text { Peduncle } \\
\text { length }\end{array}$ & $3 B$ & $\begin{array}{l}44,856 \\
819-44 \\
857,576\end{array}$ & $\begin{array}{l}\text { enzyme inhibitor } \\
\text { activity } \\
\text { (Pectinesterase inhibitor } \\
\text { domain) }\end{array}$ & $\begin{array}{l}\text { negative regulation of } \\
\text { catalytic activity }\end{array}$ \\
\hline 6 & rs61706 & $\begin{array}{l}\text { TGCAGTGGGTCGTCGGAGCATCCAAT } \\
\text { CAGATCTCCACTACACGAACGAGACTAG } \\
\text { CAGCAAGAGG_43 }\end{array}$ & $\begin{array}{l}\text { Thousand } \\
\text { kernel } \\
\text { weight }\end{array}$ & $3 B$ & $\begin{array}{l}783,413 \\
489-783 \\
414,580\end{array}$ & $\begin{array}{l}\text { GTPase activity } \\
\text { GTP binding } \\
\text { (Small GTPase AND } \\
\text { Small GTP-binding pro- } \\
\text { tein domain) }\end{array}$ & \\
\hline 7 & rs 25700 & $\begin{array}{l}\text { TGCAGCCGCTCTTCGGCGGCTCTTGCATCG } \\
\text { ATGAGCTCGCGGGTGCGGGTAAGGGGCA } \\
\text { AGTCGT_35 }\end{array}$ & $\begin{array}{l}\text { Plant } \\
\text { height }\end{array}$ & $5 B$ & $\begin{array}{l}513,646 \\
921-513 \\
649,139\end{array}$ & $\begin{array}{l}\text { catalytic activity } \\
\text { D-arabinono-1,4-lactone } \\
\text { oxidase activity } \\
\text { oxidoreductase activity } \\
\text { flavin adenine } \\
\text { dinucleotide binding } \\
\text { FAD binding }\end{array}$ & $\begin{array}{l}\text { oxidation-reduction } \\
\text { process }\end{array}$ \\
\hline 9 & rs57846 & $\begin{array}{l}\text { TGCAGTCAGAGATGATCAAGTTAAGGTCGT } \\
\text { CGAACCCGTCATGGCAGCCGCCGCCGAG } \\
\text { ATCGGA_17 }\end{array}$ & $\begin{array}{l}\text { Seed } \\
\text { number }\end{array}$ & $5 B$ & $\begin{array}{l}637,387 \\
009-637 \\
389,605\end{array}$ & $\begin{array}{l}\text { protein binding } \\
\text { (BTB/POZ domain) }\end{array}$ & \\
\hline 10 & rs46504 & $\begin{array}{l}\text { TGCAGGCATATGCTCGCCCCACATGTTCGT } \\
\text { AGACAGGCTATCCTGCCGTTACGCATTG } \\
\text { TGGTAC_30 }\end{array}$ & $\begin{array}{l}\text { Grain } \\
\text { filling } \\
\text { period }\end{array}$ & $6 B$ & $\begin{array}{l}534,921 \\
073-534 \\
927,092\end{array}$ & $\begin{array}{l}\text { guanyl-nucleotide } \\
\text { exchange factor activity } \\
\text { Rho guanyl-nucleotide } \\
\text { exchange factor activity } \\
\text { (PRONE domain AND } \\
\text { protein binding }\end{array}$ & \\
\hline 11 & rs30520 & $\begin{array}{l}\text { TGCAGCGCGACCCCTCTGCTGGCGAG } \\
\text { CTGGGTGGCCCATATATGTCTGCTTAT } \\
\text { TITATAAAAA_57 }\end{array}$ & $\begin{array}{l}\text { Days to } \\
\text { emergence }\end{array}$ & $6 \mathrm{~B}$ & $\begin{array}{l}532,043 \\
561-532 \\
045,921\end{array}$ & $\begin{array}{l}\text { anaphase-promoting } \\
\text { complex binding } \\
\text { ubiquitin-protein } \\
\text { transferase activator } \\
\text { activity }\end{array}$ & $\begin{array}{l}\text { positive regulation of } \\
\text { ubiquitin protein ligase } \\
\text { activity }\end{array}$ \\
\hline 12 & rs51526 & $\begin{array}{l}\text { TGCAGGGTACGTGAGTGATTAAACTGGCTG } \\
\text { AGTTAATTGTGATCGGCATTTGATGGTT } \\
\text { ATGGCC_47 }\end{array}$ & Grain yield & $6 \mathrm{~B}$ & $\begin{array}{l}664,500 \\
180-664 \\
501,715\end{array}$ & - & $\begin{array}{l}\text { asymmetric cell } \\
\text { division }\end{array}$ \\
\hline 13 & rs56337 & $\begin{array}{l}\text { TGCAGTACCGCTCTTCCCGAGCTGGCACTA } \\
\text { CTGTTCCACCCGTCCAACGATCTGTTGG } \\
\text { GGCATC_32 }\end{array}$ & Grain yield & $7 \mathrm{~A}$ & $\begin{array}{l}80,142 \\
837-80 \\
144,941\end{array}$ & $\begin{array}{l}\text { galactoside 2-alpha-L- } \\
\text { fucosyltransferase } \\
\text { activity } \\
\text { (Xyloglucan } \\
\text { fucosyltransferase }\end{array}$ & $\begin{array}{l}\text { fucosylation } \\
\text { cell wall biogenesis }\end{array}$ \\
\hline 14 & rs53016 & $\begin{array}{l}\text { TGCAGGTCCCATGGCCTCTACCATAGTCGA } \\
\text { ACGGAGGTGGATGCGCTTTGAGGTGGAT } \\
\text { GCCTGA_35 }\end{array}$ & $\begin{array}{l}\text { Grain } \\
\text { filling } \\
\text { period }\end{array}$ & $7 \mathrm{~B}$ & $\begin{array}{l}15,713 \\
548-15 \\
714,633\end{array}$ & $\begin{array}{l}\text { DNA binding } \\
\text { DNA-binding } \\
\text { transcription factor } \\
\text { activity } \\
\text { (NA-binding domain } \\
\text { superfamily, AP2/ERF } \\
\text { domain) }\end{array}$ & $\begin{array}{l}\text { regulation of } \\
\text { transcription, DNA- } \\
\text { templated }\end{array}$ \\
\hline
\end{tabular}


Table 7 Description of expected MTAs using imputed SNPs for agronomic traits of Iranian wheat accessions exposed to the rain-fed conditions

\begin{tabular}{|c|c|c|c|c|c|c|c|}
\hline Row & Marker & sequence & Trait & Chromosome & $\begin{array}{l}\text { Position } \\
\text { (bp) }\end{array}$ & Molecular process & Biological process \\
\hline 1 & rs64750 & $\begin{array}{l}\text { TGCAGTTATGTACGAACTITGAGAA } \\
\text { TCTCATCAGTGGCCAAACGCCCAAACT } \\
\text { AACAATTGAA_34 }\end{array}$ & Canopy temperature & $4 \mathrm{~A}$ & $\begin{array}{l}\text { 630,897, } \\
051-630 \\
899,273\end{array}$ & $\begin{array}{l}\text { DNA binding } \\
\text { (3 DNA binding } \\
\text { domain) }\end{array}$ & $\begin{array}{l}\text { transcription, DNA- } \\
\text { templated } \\
\text { regulation of } \\
\text { transcription, DNA- } \\
\text { templated }\end{array}$ \\
\hline 2 & rs11116 & $\begin{array}{l}\text { TGCAGCAAATTAATCTAGCTTTIAGT } \\
\text { TICCTICAGGTATITGGATATGCCAGC } \\
\text { AAATCGAAA__29 }\end{array}$ & Peduncle length & $4 \mathrm{~A}$ & $\begin{array}{l}739,791 \\
132-739 \\
802,785\end{array}$ & $\begin{array}{l}\text { ADP binding } \\
\text { (NB-ARC) }\end{array}$ & - \\
\hline 3 & rs736 & $\begin{array}{l}\text { TGCAGAAAGGTACCACTCATTCGTAC } \\
\text { ATCACTCCAACTGATGTATGAAGGTTGT } \\
\text { TCATGGCGAC_18 }\end{array}$ & Spike weight & $4 \mathrm{~B}$ & $\begin{array}{l}481,233 \\
765-481 \\
237,258\end{array}$ & hydrolase activity & $\begin{array}{l}\text { phosphatidylinositol } \\
\text { dephosphorylation }\end{array}$ \\
\hline 4 & rs55557 & $\begin{array}{l}\text { TGCAGGTTITGCCTAAGAAAAACTCA } \\
\text { GAATTCACTCAAAAAAATCAGATTGCTG } \\
\text { TAAACTGCAC_15 }\end{array}$ & Canopy temperature & $4 \mathrm{~B}$ & $\begin{array}{l}613,031 \\
990-613 \\
041,407\end{array}$ & $\begin{array}{l}\text { drug transmembrane } \\
\text { transporter activity } \\
\text { antiporter activity } \\
\text { (Multi antimicrobial } \\
\text { extrusion protein) }\end{array}$ & $\begin{array}{l}\text { drug } \\
\text { transmembrane } \\
\text { transport } \\
\text { transmembrane } \\
\text { transport }\end{array}$ \\
\hline 5 & rs50187 & $\begin{array}{l}\text { TGCAGGGCAGTCGAAGCAGTTGCTGG } \\
\text { GTCAGAGGCGTGGAGTTGCACTGG } \\
\text { AGCAACAGGAGTCG_54 }\end{array}$ & Spike length & $4 \mathrm{~B}$ & $\begin{array}{l}222,603 \\
782-222 \\
615,097\end{array}$ & $\begin{array}{l}\text { transmembrane } \\
\text { transporter activity } \\
\text { (Major facilitator, } \\
\text { sugar transporter-like) }\end{array}$ & $\begin{array}{l}\text { transmembrane } \\
\text { transport }\end{array}$ \\
\hline 6 & rs41689 & $\begin{array}{l}\text { TGCAGCTTGTCGGTCCTCTCCGACAT } \\
\text { GGCGTCGAGCACCCGCCGAGTCTG } \\
\text { GGCCGAGGGTTTG__15 }\end{array}$ & Leaf greenness & $5 B$ & $\begin{array}{l}334,871 \\
156-334 \\
874,981\end{array}$ & $\begin{array}{l}\text { catalytic activity } \\
\text { ATP binding } \\
\text { zinc ion binding } \\
\text { pyridoxal phosphate } \\
\text { binding } \\
\text { cysteine desulfurase } \\
\text { activity } \\
\text { (Cysteine desulfurase } \\
\text { IscS) }\end{array}$ & $\begin{array}{l}\text { iron-sulfur cluster } \\
\text { assembly } \\
\text { [2Fe-2S] cluster } \\
\text { assembly }\end{array}$ \\
\hline 7 & rs59282 & $\begin{array}{l}\text { TGCAGTCGTGGATAATGCACCTTGCG } \\
\text { GTGTCAGGGGGTGACGTCAGCGAT } \\
\text { GAGTCCACCG_39 }\end{array}$ & Days to heading & $5 B$ & $\begin{array}{l}11,550 \\
484-11 \\
556,238\end{array}$ & $\begin{array}{l}\text { catalytic activity } \\
\text { hydrolase activity, } \\
\text { hydrolyzing O- } \\
\text { glycosyl compounds } \\
\text { alpha-galactosidase } \\
\text { activity } \\
\text { hydrolase activity } \\
\text { hydrolase activity, } \\
\text { acting on glycosyl } \\
\text { bonds } \\
\text { raffinose alpha- } \\
\text { galactosidase activity } \\
\text { (Glycoside hydrolase, } \\
\text { family 27) }\end{array}$ & $\begin{array}{l}\text { carbohydrate } \\
\text { metabolic process } \\
\text { metabolic process }\end{array}$ \\
\hline 8 & rs44154 & $\begin{array}{l}\text { TGCAGGAGCACCAGCGCGGCAGCGGT } \\
\text { GGCGACGACGGGGCTACCAGCTGC } \\
\text { CCGCCGAGATCGGA_20 }\end{array}$ & Spike length & $5 B$ & $\begin{array}{l}363,662 \\
473-363 \\
670,095\end{array}$ & $\begin{array}{l}\text { catalytic activity } \\
\text { hydrolase activity, } \\
\text { hydrolyzing O- } \\
\text { glycosyl compounds } \\
\text { cellulase activity } \\
\text { hydrolase activity } \\
\text { hydrolase activity, } \\
\text { acting on glycosyl } \\
\text { bonds }\end{array}$ & $\begin{array}{l}\text { polysaccharide } \\
\text { catabolic process } \\
\text { carbohydrate } \\
\text { metabolic process } \\
\text { metabolic process } \\
\text { cellulose catabolic } \\
\text { process }\end{array}$ \\
\hline 9 & rs17806 & $\begin{array}{l}\text { TGCAGCAGGCAAGGTATCTCCAGGCG } \\
\text { AACTATATCATCGCAATATACGAGCTTC } \\
\text { AGGTGCTCCA_61 }\end{array}$ & $\begin{array}{l}\text { Days to heading, } \\
\text { anthsis and } \\
\text { physiological } \\
\text { maturity }\end{array}$ & $5 B$ & $\begin{array}{l}457,966 \\
329-457 \\
970,659\end{array}$ & $\begin{array}{l}\text { protein binding } \\
\text { (F-box-like domain } \\
\text { superfamily) }\end{array}$ & - \\
\hline 10 & rs 25700 & $\begin{array}{l}\text { TGCAGCCGCTCTTCGGCGGCTCTTGC } \\
\text { ATCGATGAGCTCGCGGGTGCGGGT } \\
\text { AAGGGGCAAGTCGT_35 }\end{array}$ & Peduncle length & $5 B$ & $\begin{array}{l}513,646 \\
921-513 \\
649,139\end{array}$ & $\begin{array}{l}\text { catalytic activity } \\
\text { D-arabinono-1,4- } \\
\text { lactone oxidase } \\
\text { activity } \\
\text { oxidoreductase }\end{array}$ & $\begin{array}{l}\text { oxidation-reduction } \\
\text { process }\end{array}$ \\
\hline
\end{tabular}


Table 7 Description of expected MTAs using imputed SNPs for agronomic traits of Iranian wheat accessions exposed to the rain-fed conditions (Continued)

\begin{tabular}{|c|c|c|c|c|c|c|}
\hline Row Marker & sequence & Trait & Chromosome & $\begin{array}{l}\text { Position } \\
\text { (bp) }\end{array}$ & Molecular process & Biological process \\
\hline & & & & & $\begin{array}{l}\text { activity } \\
\text { flavin adenine } \\
\text { dinucleotide binding } \\
\text { FAD binding }\end{array}$ & \\
\hline
\end{tabular}

The SNPs rs48893, rs10316, and rs36032 all show relatively high variance explained for the associated traits.

As illustrated in Table 9, under rain-fed conditions, 3, $2,2,2,1$, and 1 of the original SNPs were positively associated to spike weight (by 0.02, 0.03, and $0.03 \mathrm{~g}$ ), thousand kernel weight (by 1.59 , and $1.90 \mathrm{~g}$ ), grain yield (by 0.03 and $0.03 \mathrm{~g}$ ), and grain filling period (by 1.31, and $1.57 \mathrm{~d}$ ), seed number per spike (by 0.58), and leaf greenness (by 1.69). In contrast, rs2234, rs25700, rs46075, and rs30520 had positive effects on spike length (by $0.53 \mathrm{~cm}$ ), plant height (by $11.06 \mathrm{~cm}$ ), peduncle length (by $1.76 \mathrm{~cm}$ ), and days to emergence (by $0.28 \mathrm{~d}$ ). However, the number of favorable alleles for spike length, spike weight, peduncle length, days to heading, days to anthesis, days to physiological maturity, canopy temperature and leaf greenness were $2,1,2$, $2,1,1,2$, and 1, respectively. Rs11116 and rs25700 decreased peduncle length by 2.66 and $4.56 \mathrm{~cm}$, rs59282 and rs17806 also decreased days to heading, anthesis, and maturity by $4.24,7.59,7.04$, and $6.78 \mathrm{~d}$ and rs64750 caused a decline in canopy temperature by $2.61^{\circ} \mathrm{C}$. Under rain-fed conditions, PVE ranged from 7 to $38 \%$. SNPs associated with grain yield, spike weight and seed number all explained a considerable proportion of the phenotypic variance. Moreover, the SNP rs17806 adjusted days to anthesis and physiological maturity by about 36 and 38\%, respectively. Manhattan and QQ-plots of highly associated haplotypes for agronomic traits are shown in Fig. 6.

\section{Discussion}

Improving wheat grain yield is a high priority of wheat breeders in order to meet increasing demands worldwide. In the current study, we have explored the diversity of the Iranian hexaploid wheat population and performed association mapping studies for a number of important agronomic traits. These traits influence grain yield either directly or indirectly under well-watered or rain-fed conditions. Significant positive or negative relationships were observed among these traits, which can be used to gauge their impact on target traits like grain yield and grain yield-related attributes. Given that most agronomic traits are polygenic and drought tolerance is a complex mechanism involving many pathways, we focused on three categories of agronomic traits employing a large diversity panel. Furthermore, using a diverse gene pool help increase the resolution of association mapping. We, therefore, tested both historical and modern varieties as well as representative landraces from different climate zones in order to include sufficient genetic variation to be able to map trait-relevant variation.

Most Iranian varieties originate from International Maize and Wheat Improvement Center (CIMMYT)

Table 8 The effect of favorable alleles on agronomic traits of Iranian wheat accessions exposed to the well-watered conditions

\begin{tabular}{|c|c|c|c|c|c|c|c|c|c|}
\hline SNPs & Trait & Marker & $a i$ & Typical accession & Allele & Favorable allele & MAF & $p(-\log 10)$ & $R^{2}$ \\
\hline \multirow[t]{4}{*}{ Original } & $\mathrm{CT}$ & rs62576 & -0.72 & 623,266 & $A / G$ & $A$ & 0.14 & 3.68 & 0.40 \\
\hline & SN & $\begin{array}{l}\text { rs } 48893 \\
\text { rs10316 }\end{array}$ & $\begin{array}{l}0.96 \\
1.20\end{array}$ & 624,251 & $\begin{array}{l}C / T \\
C / T\end{array}$ & $\begin{array}{l}\mathrm{T} \\
\mathrm{C}\end{array}$ & $\begin{array}{l}0.32 \\
0.14\end{array}$ & $\begin{array}{l}3.51 \\
3.59\end{array}$ & $\begin{array}{l}0.24 \\
0.2 C\end{array}$ \\
\hline & TKW & rs4772 & 0.77 & 623,345 & $A / C$ & C & 0.43 & 3.23 & 0.11 \\
\hline & GFP & rs46504 & 0.90 & 626,158 & $A / C$ & C & 0.19 & 4.30 & 0.13 \\
\hline \multirow[t]{5}{*}{ Imputed } & GY & rs36032 & 0.01 & 622,098 & $C / T$ & C & 0.10 & 3.16 & 0.21 \\
\hline & SW & $\begin{array}{l}\text { rs36032 } \\
\text { rs } 736\end{array}$ & $\begin{array}{l}0.02 \\
0.33\end{array}$ & $\begin{array}{l}622,098 \\
\text { Neishabour }\end{array}$ & $\begin{array}{l}C / T \\
A / G\end{array}$ & $\begin{array}{l}C \\
G\end{array}$ & $\begin{array}{l}0.10 \\
0.11\end{array}$ & $\begin{array}{l}3.16 \\
3.56\end{array}$ & $\begin{array}{l}0.21 \\
0.10\end{array}$ \\
\hline & SL & $\begin{array}{l}\text { rs34314 } \\
\text { rs40819 } \\
\text { rs36808 }\end{array}$ & $\begin{array}{l}-1.09 \\
-0.70 \\
-0.50\end{array}$ & $\begin{array}{l}\text { Neishabour } \\
\text { Chamran } \\
621,650\end{array}$ & $\begin{array}{l}C / T \\
G / A \\
A / G\end{array}$ & $\begin{array}{l}\mathrm{T} \\
\mathrm{A} \\
\mathrm{G}\end{array}$ & $\begin{array}{l}0.04 \\
0.06 \\
0.39\end{array}$ & $\begin{array}{l}4.71 \\
4.29 \\
3.41\end{array}$ & $\begin{array}{l}0.12 \\
0.12 \\
0.10\end{array}$ \\
\hline & $\mathrm{DE}$ & rs57386 & -0.11 & 625,081 & $\mathrm{~A} / \mathrm{C}$ & A & 0.22 & 3.61 & 0.07 \\
\hline & LG & rs34075 & 1.82 & 627,852 & $\mathrm{~A} / \mathrm{C}$ & $A$ & 0.26 & 3.88 & 0.15 \\
\hline
\end{tabular}


Table 9 The effect of favorable alleles on agronomic traits of Iranian wheat accessions exposed to the rain-fed conditions

\begin{tabular}{|c|c|c|c|c|c|c|c|c|c|}
\hline SNPS & Trait & Marker & $a i$ & Typical accession & Allele & Favorable allele & MAF & $p(-\log 10)$ & $R^{2}$ \\
\hline \multirow[t]{10}{*}{ Original } & SW & $\begin{array}{l}\text { rs63808 } \\
\text { rs15903 } \\
\text { rs51526 }\end{array}$ & $\begin{array}{l}0.02 \\
0.03 \\
0.03\end{array}$ & $\begin{array}{l}623,125 \\
\text { BAHAR } \\
623,125\end{array}$ & $\begin{array}{l}A / G \\
A / G \\
A / G\end{array}$ & $\begin{array}{l}G \\
A \\
A\end{array}$ & $\begin{array}{l}0.15 \\
0.32 \\
0.19\end{array}$ & $\begin{array}{l}3.60 \\
3.47 \\
3.26\end{array}$ & $\begin{array}{l}0.32 \\
0.32 \\
0.32\end{array}$ \\
\hline & SL & rs2237 & -0.53 & 627,057 & $A / G$ & G & 0.17 & 4.62 & 0.13 \\
\hline & TKW & $\begin{array}{l}\text { rs26577 } \\
\text { rs61706 }\end{array}$ & $\begin{array}{l}1.57 \\
1.90\end{array}$ & $\begin{array}{l}625,123 \\
623,909\end{array}$ & $\begin{array}{l}C / T \\
A / G\end{array}$ & $\begin{array}{l}T \\
G\end{array}$ & $\begin{array}{l}0.48 \\
0.21\end{array}$ & $\begin{array}{l}3.57 \\
3.82\end{array}$ & $\begin{array}{l}0.09 \\
0.09\end{array}$ \\
\hline & SN & rs57846 & 0.58 & Bahar & $\mathrm{A} / \mathrm{T}$ & T & 0.22 & 3.43 & 0.37 \\
\hline & GY & $\begin{array}{l}\text { rs51526 } \\
\text { rs56337 }\end{array}$ & $\begin{array}{l}0.03 \\
0.03\end{array}$ & $\begin{array}{l}627,410 \\
625,047\end{array}$ & $\begin{array}{l}A / G \\
G / T\end{array}$ & $\begin{array}{l}A \\
G\end{array}$ & $\begin{array}{l}0.19 \\
0.25\end{array}$ & $\begin{array}{l}3.44 \\
3.91\end{array}$ & $\begin{array}{l}0.21 \\
0.22\end{array}$ \\
\hline & $\mathrm{PH}$ & rs 25700 & -11.06 & 623,318 & $C / G$ & G & 0.14 & 3.93 & 0.24 \\
\hline & $P L$ & rs46075 & -1.76 & 623,139 & $C / T$ & C & 0.19 & 3.51 & 0.09 \\
\hline & GFP & $\begin{array}{l}\text { rs46504 } \\
\text { rs53016 }\end{array}$ & $\begin{array}{l}1.31 \\
1.57\end{array}$ & $\begin{array}{l}626,360 \\
623,905\end{array}$ & $\begin{array}{l}A / C \\
G / T\end{array}$ & $\begin{array}{l}C \\
T\end{array}$ & $\begin{array}{l}0.19 \\
0.16\end{array}$ & $\begin{array}{l}3.63 \\
5.21\end{array}$ & $\begin{array}{l}0.09 \\
0.11\end{array}$ \\
\hline & DE & rs30520 & -0.28 & 626,825 & $A / G$ & G & 0.13 & 3.41 & 0.07 \\
\hline & LG & rs33549 & 1.69 & Gascogne & $A / G$ & G & 0.19 & 3.65 & 0.10 \\
\hline \multirow[t]{8}{*}{ Imputed } & SL & $\begin{array}{l}\text { rs50187 } \\
\text { rs44154 }\end{array}$ & $\begin{array}{l}-0.39 \\
-0.14\end{array}$ & $\begin{array}{l}626,924 \\
627,057\end{array}$ & $\begin{array}{l}A / G \\
A / G\end{array}$ & $\begin{array}{l}A \\
G\end{array}$ & $\begin{array}{l}0.22 \\
0.21\end{array}$ & $\begin{array}{l}4.04 \\
3.42\end{array}$ & $\begin{array}{l}0.14 \\
0.13\end{array}$ \\
\hline & SW & rs736 & 0.29 & Alvand & $A / G$ & G & 0.11 & 3.81 & 0.32 \\
\hline & $P L$ & $\begin{array}{l}\text { rs11116 } \\
\text { rs25700 }\end{array}$ & $\begin{array}{l}-2.66 \\
-4.56\end{array}$ & $\begin{array}{l}627,410 \\
623,344\end{array}$ & $\begin{array}{l}C / T \\
C / G\end{array}$ & $\begin{array}{l}C \\
G\end{array}$ & $\begin{array}{l}0.12 \\
0.11\end{array}$ & $\begin{array}{l}3.55 \\
3.65\end{array}$ & $\begin{array}{l}0.08 \\
0.08\end{array}$ \\
\hline & $\mathrm{DH}$ & $\begin{array}{l}\text { rs59282 } \\
\text { rs17806 }\end{array}$ & $\begin{array}{l}-4.24 \\
-7.59\end{array}$ & $\begin{array}{l}\text { Dastjerdi } \\
\text { Kavir } \\
624,818\end{array}$ & $\begin{array}{l}\mathrm{A} / \mathrm{C} \\
\mathrm{C} / \mathrm{T}\end{array}$ & $\begin{array}{l}C \\
T\end{array}$ & $\begin{array}{l}0.23 \\
0.11\end{array}$ & $\begin{array}{l}3.45 \\
3.69\end{array}$ & $\begin{array}{l}0.36 \\
0.36\end{array}$ \\
\hline & DA & rs17806 & -7.04 & $\begin{array}{l}\text { Shanghai7 } \\
\text { Kavir }\end{array}$ & $\mathrm{C} / \mathrm{T}$ & $\mathrm{T}$ & 0.11 & 3.30 & 0.38 \\
\hline & DM & rs17806 & -6.78 & $\begin{array}{l}\text { Frontana } \\
624,818\end{array}$ & $\mathrm{C} / \mathrm{T}$ & T & 0.11 & 3.44 & 0.32 \\
\hline & $\mathrm{CT}$ & $\begin{array}{l}\text { rs64750 } \\
\text { rs55557 }\end{array}$ & $\begin{array}{l}-2.61 \\
-0.39\end{array}$ & $\begin{array}{l}624,315 \\
622,105\end{array}$ & $\begin{array}{l}A / G \\
A / T\end{array}$ & $\begin{array}{l}G \\
T\end{array}$ & $\begin{array}{l}0.03 \\
0.49\end{array}$ & $\begin{array}{l}4.32 \\
3.58\end{array}$ & $\begin{array}{l}0.11 \\
0.09\end{array}$ \\
\hline & LG & rs41689 & 0.82 & $\begin{array}{l}\text { Roshan } \\
623,344\end{array}$ & $C / G$ & $C$ & 0.24 & 3.83 & 0.12 \\
\hline
\end{tabular}

materials with twenty-four varieties in advanced and segregating lines, originating from this center. At least, forty varieties were obtained through a cross-breeding program where one of the CIMMYT advanced lines was one of the parents (Additional file 1: Table S1). A large number of these varieties were released after green revolution. A previous study on historical wheat varieties from Pakistan illustrated the considerable contribution of CIMMYT germplasm, which has been used in the development of Iranian varieties as well [21]. We identified three clusters of varieties with mixed genetic backgrounds with no clear relation to the release year. The relatively small number of the varieties derived from Iran suggests a relatively narrow utilization of Iranian landraces among the current and old varieties, which could be a substantial genetic bottleneck.

In line with earlier studies, we identified most SNPs in the $\mathrm{B}$ and $\mathrm{A}$ genomes whereas the younger $\mathrm{D}$ genome showed a lower number of SNPs [32,33]. We observed the same trend also for the number of marker pairs in
$\mathrm{LD}$, where SNPs mapping to the $\mathrm{B}$ genome were approximately three times more common than those mapping to the $\mathrm{D}$ genome. The most significant marker pairs were observed on chromosomes $2 \mathrm{~B}$ and $3 \mathrm{~B}$ in both, varieties and landraces (Table 2). The higher diversity seen in the A and B genomes could be the result of their older evolutionary background and due to gene flow from T. turgidum as opposed to lack of gene flow from Ae. tauschii to bread wheat [34, 35]. Moreover, a bottleneck effect likely occurred due to strong selection among ancestral hexaploid landraces in modern varieties breeding programs and this may have further effects on the D genome. Such a bottleneck would result in a reduction in the effective population size, which increases the rate of loss of low-frequency alleles in the A and B genomes, and on the other hand, a higher proportion of rare alleles in the $\mathrm{D}$ genome indicate a reduction in the allelic variant for younger genome [36]. Our results show that most markers that are in significant LD are located at distances $<10 \mathrm{cM}$. However, LD and marker 

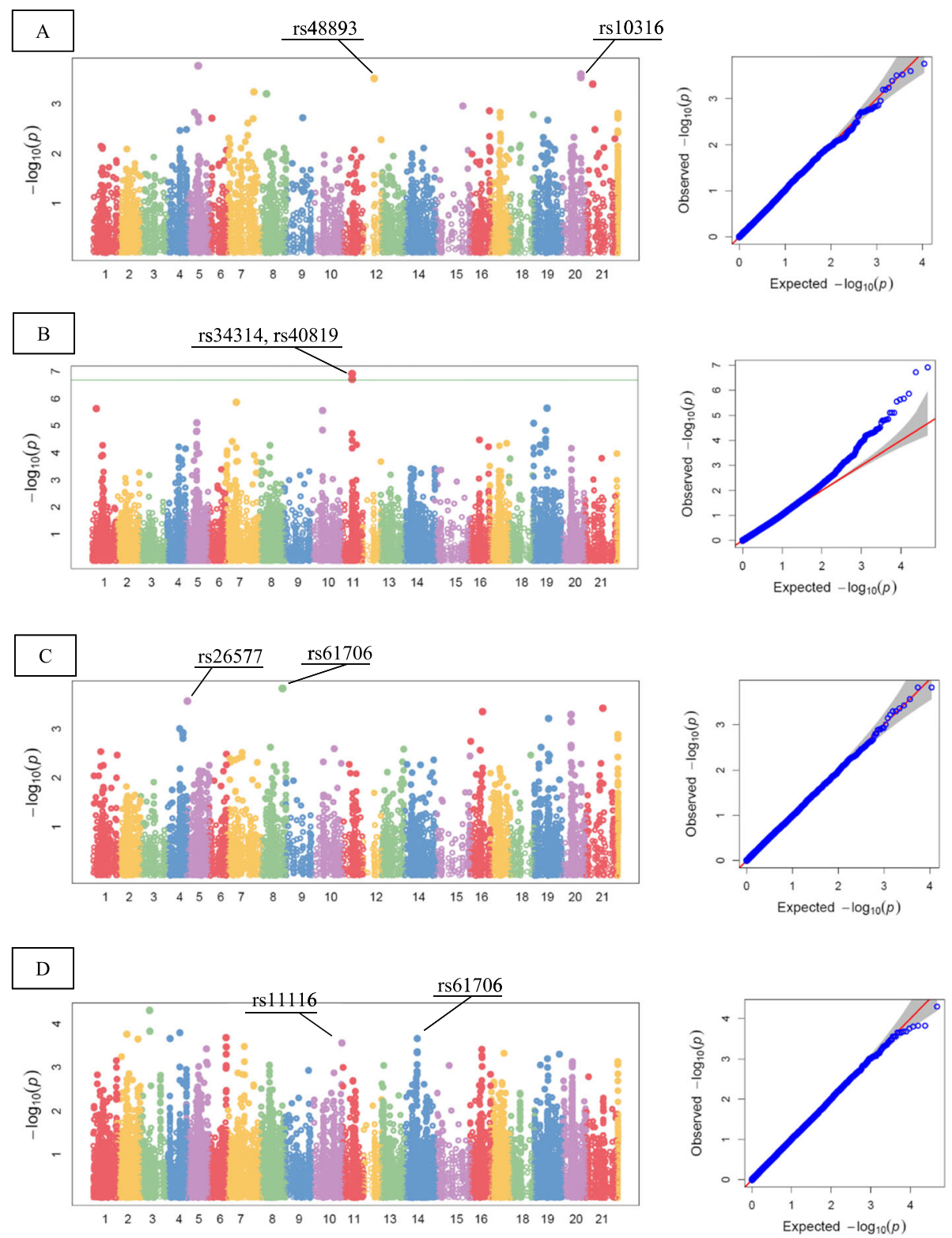

Fig. 6 Manhattan and QQ-plots of highly associated haplotypes for agronomic traits under well-watered and rain-fed conditions. A) seed number per spike, B) spike length, $C$ ) thousand kernel weight, $D$ ) peduncle length. $X$ axis represents chromosomes: 1)1 $A, 2) 1 B, 3) 1 D, 4) 2 A, 5) 2 B, 6) 2 D$, 7) $3 A$, 8) $3 B$, 9) $3 D, 10) 4 A, 11) 4 B, 12) 4 D, 13) 5 A, 14) 5 B, 15) 5 D, 16) 6 A, 17) 6 B, 18) 6 D, 19) 7 A, 20) 7 B, 21) 7 D$

distances across the $\mathrm{D}$ genome were much higher than in the other two sub-genomes. The greater extent of linkage across all genomes in varieties clearly indicates the effect of selection during the history of those accessions (Table 2). Selection, recombination, mutation, genetic drift, mating systems and population relatedness are all major factors, which influence linkage disequilibrium [37-39]. The fact that varieties show significantly overall higher LD compared to landraces, in particular in the D genome, is likely the result of selection during breeding for important agronomic traits [40, 41].
While mapping traits with low heritability may not result in a desirable gain, utilizing highly correlated traits can increase power and therefore help with the rapid advancement of breeding programs. Although grain yield is the most reliable selection criterion in different target environments, particularly for water deficit conditions, the complex genetic architecture of this trait has thus far limited direct genome-based selection. However, the pleiotropic effect of genes controlling this trait and the close connection between grain yield and drought tolerance mechanisms highlights the role that other 
morphological, phenological and physiological traits play and which should be considered in any selection strategy.

Among 313 and 394 identified MTAs for the original and imputed SNPs, respectively, 86 and 76 falls within coding genes with $P$-values $<0.001$. To eliminate possible false-positive associations, we selected the most strongly associated markers which yielded 19 and 17 markers located across all chromosomes and were identified in both environments using the two SNP datasets. Using the original SNPs under well-watered conditions, we identified QTLs for canopy temperature (1A), seed number (4D, and $7 \mathrm{~B})$, thousand kernel weight $(5 \mathrm{~B})$, and grain filling period (6B). Using the imputed SNPs, we observed QTLs for grain yield and spike weight on chromosomes $1 \mathrm{~A}$ and $4 \mathrm{~B}$, for leaf greenness on chromosome $1 \mathrm{~A}$, three QTLs on $4 \mathrm{~B}$ and $5 \mathrm{~B}$ for spike length and one QTL on $5 \mathrm{~A}$ for days to emergence. These results are in line with previously detected QTLs for spike length [42], grain yield [42], seed number per spike [43] and thousand kernel weight [44].

For rain-fed conditions, we identified QTLs on chromosomes $1 \mathrm{~B}, 2 \mathrm{~B}$ for spike weight, $6 \mathrm{~B}$ and $7 \mathrm{~A}$ for grain yield, $1 \mathrm{~B}$ for spike length, $2 \mathrm{~B}$ and $3 \mathrm{~B}$ for thousand kernel weight, $3 \mathrm{~B}$ for peduncle length, $5 \mathrm{~B}$ for plant height, $5 \mathrm{~B}$ for leaf greenness, $5 \mathrm{~B}$ for seed number per spike, $6 \mathrm{~B}$ and $7 \mathrm{~B}$ for grain filling period and $6 \mathrm{~B}$ for days to emergence. These results are in line with findings by Ain et al. [21] for plant height, thousand kernel weight and grain yield. Bossolini et al. [45] and Acuna-Galindo et al. [46] identified stable QTLs on chromosomes 6B and $5 \mathrm{~B}$ for grain yield and plant height, respectively. MTAs for seed number per spike on chromosome $5 \mathrm{~B}$ have been previously reported $[47,48]$. Neumann et al. [14] reported a QTL on chromosome 2B for spike weight. Using imputed SNPs and phenotypic data under rain-fed conditions, all identified MTAs were located on chromosomes $4 \mathrm{~A}, 4 \mathrm{~B}$ and $5 \mathrm{~B}$, with QTLs for canopy temperature (4A and $4 \mathrm{~B})$, peduncle length (4A, and $5 B$ ), spike weight (4B), spike length (4B and $5 B$ ), leaf greenness $(5 \mathrm{~B})$ and days to heading, anthesis, maturity $(5 \mathrm{~B})$. These results are in agreement with previously reported QTLs for these traits [14, 33, 47, 48]. In addition, we also identified a number of novel chromosomal regions that harbored MTAs for physiological parameters and phenological growth stages. For instance, under well-watered conditions, rs62576 (1A), rs34075 (1A) and rs57386 (5A) were associated with canopy temperature, leaf greenness and days to emergence. Under rain-fed conditions, markers rs64750 (4A) and rs55557 (4B) were associated with canopy temperature whereas rs4607 (3B) and rs41689 (5B) were associated with peduncle length and leaf greenness, respectively. Moreover, marker rs17806 (5B) has pleiotropic effects on days to heading, anthesis, and physiological maturity.

Although imputation of missing data significantly increased the potential number of MTAs, mainly on the A and B genomes, the fraction of SNPs presents within coding genes declined, from about $27 \%$ in the original SNP data set to $19.6 \%$ for the imputed dataset. This suggests that most SNPs with missing data are located in noncoding DNA regions where read mapping and SNP calling are known to be more problematic in most plant genomes. Dissecting strongly associated chromosomal regions through, for instance, positional cloning to identify putative causal genes is the next logical step following association mapping studies. Apart from using comparative genomics approaches to identify the function of associated genes, independent functional validation is also required to guarantee the success of either positional cloning or transgenic experiments [21, 4951]. We obtained the flanking sequence of putative SNPs and aligned them against the IWGSC RefSeq v1.0. This information showed that most genes identified through the association study are involved in important biosynthesis pathways such as oxidation-reduction, carbohydrate metabolism, ion transportation and cell wall biogenesis. The protein encoded by these genes are mostly involved in DNA-binding, ATP-binding, peroxidase activity, protein kinase activity, metal ion binding, enzyme inhibitor activity, serine-type endopeptidase activity, hydrolase activity, antiporter activity and transmembrane activity. Such associations have also been reported in earlier research [52-58]. These genes are all located in chromosomal regions, which show a strong association with important agronomic traits and they can thus be considered as suitable target genes for future breeding programs. We calculated the phenotypic effect of favorable alleles, as described by Dong et al. [59] to show that they affected grain yield only slightly but had much larger effect on thousand kernel weight, spike length and leaf greenness. On the other hand, alleles that contribute to an improvement in thousand kernel weight and leaf greenness also have pleiotropic effects thereby decreasing plant height, peduncle length and canopy temperature under stressful conditions. Most identified MTAs exist across genes which are involved in multilayer processes and complex networks, therefore their minor impact on agronomic traits is not too far-fetched.

\section{Conclusions}

In the present study, GWAS was performed for important agronomic traits of bread wheat in a diverse panel of 298 varieties and landraces of Iran collection. The highest number of marker pairs in both varieties and landraces was observed on B genome. In total, 313 and 394 MTAs were identified for 14 phenological, agronomic 
and physiological traits using original and imputed SNPs, respectively. The identified association between markers and traits generally lied in a range of $10^{-3}$ and $10^{-4}$. It seems that complex inheritance of such quantitative traits and high number of controlling genes exclude greater association. However, a major part of found MTAs explained more than $20 \%$ of total phenotypic variation for relevant traits. Although, further studies are required to validate the detected markers in this study using other populations and environments. Gene ontology of identified markers in original and imputed SNPs showed approximately $27 \%$ of these markers represent within coding genes, thereby have potential to be used in genome-based breeding of new varieties. Although imputation of missing data could increase the number of associated markers, the percentage of MTAs located in coding regions was decreased across different sub-genomes. The identified markers in this study could provide useful genetic resources to initiate markerassisted selection, fine mapping and cloning of the underlying genes and QTLs.

\section{Methods}

\section{Plant material and experiment conditions}

A set of 320 Iranian wheat accession, including 102 varieties released between 1942 and 2014, and 218 landraces collected between 1931 and 1968 (Additional file 1: Table S1) were tested under a well-watered system and rain-fed conditions using an alpha-lattice design with two replicates at the agricultural research lands of the Department of Agronomy and Plant Breeding, University of Tehran. Plant materials were kindly provided by the University of Tehran and Seed and Plant Improvement Institute (SPII), Karaj, Iran. Both phenotypic and genotypic data were available for 298 accessions (90 varieties and 208 landraces). The field site is located at $\mathrm{N} 35^{\prime} .80^{\circ}$ and $\mathrm{E} 50^{\prime} .95^{\circ}$ in Karaj, Iran, and experiments were conducted during the cropping seasons of 2016-17 and 2017-18 (weather conditions are given in Additional file 3: Fig. S1).

\section{Field trial}

Plant development was scored according to the Zadoks scale and included i) days to emergence (Zadoks 12), ii) days to heading (Zadoks 50), iii) days to anthesis (Zadoks 65), iv) days to physiological maturity (Zadoks 91), and v) grain filling period when half of each plot had reached to corresponding stages. The Soil Plant Analysis Development (SPAD, Minolta Camera Co., Osaka, Japan, SPAD502 Plus Chlorophyll Meter) and LIHERO Infrared thermometer were used to measure leaf greenness and canopy temperature at Zadoks 60, respectively. Grain yield and related traits including spike weight, spike length, seed number per spike and thousand kernel weight were measured after harvesting for both years.

\section{Genotyping by sequencing and imputation method}

The development and sequencing of a GBS library for the Iranian wheat have previously been described by Alipour et al. [30]. Briefly, after trimming sequencing reads to $64 \mathrm{bp}$ and grouping them into sequence tags, SNPs were identified using internal alignment allowing for mismatch up to $3 \mathrm{bp}$. The UNEAK (Universal NetworkEnabled Analysis Kit) GBS pipeline was used for SNPs calling, where reads with a low-quality score $(<15)$ and SNPs with low minor allele frequency $<1 \%$ were removed to avoid false-positive markers arising from sequencing errors. The data was also subjected to imputation using BEAGLE v3.3.2 [60] based on available allele frequencies obtained after specifying the haplotype phase for all individuals. Four different reference genomes were assessed during imputation and W7984 reference genome was shown to have the greatest imputation accuracy [31]. The LD decay of different chromosomes was obtained using the ggplot2 package in RStudio [61] based on LOESS regression.

\section{Population structure and kinship matrix}

Population structure in the sample was estimated using STRUCTURE v.2.3.4 [62] with an admixture model and with a burn-in and simulation phase consisting of 10,000 steps for values of $K=1$ to 10 . $\Delta \mathrm{K}$ was plotted for consecutive $\mathrm{K}$ values and used to determine the most likely number of subpopulations. The values of observed and expected allele frequencies were used to calculate LD among markers in TASSEL v.5 [63]. A structure matrix (Q-matrix) was then obtained for all accessions used for association studies. To determine the relationship between varieties and landraces, a neighbor-joining tree was constructed based on a pairwise distance matrix calculated in TASSEL v.5 [63] and visualized using Archaeopteryx (https://sites.google.com/site/cmzmasek/home/ software/archaeopteryx).

\section{Genome-wide association study}

We used both general linear model (GLM) and mixed linear model (MLM) to obtain the unbiased estimation of marker effects. The MLM approach resulted in the most accurate association of marker-traits and different versions of the MLM model, including Q, K or Q + K, were used to control both effects of population structure $(\mathrm{Q})$ and more diffused relationships (K) among accessions using TASSEL v.5 [63]. The GAPIT package [64] was used to perform association mapping for the MLM model in RStudio [61]. Results from both TASSEL [63] and GAPIT [64] evaluated based on the significance of associated loci using $t$-tests. In general, GAPIT [64] provided a stronger control 
of confounding effects. We, therefore, only reported results from GAPIT [64]. In the MLM model, individuals are considered as random effects and the relatedness among individuals is conveyed through a kinship matrix. To perform cluster analysis, kinship matrix elements were used as similarity measures and clusters visualized using unweighted pair group method with arithmetic mean (UPGMA) through the heat map plot. A Manhattan plot is a visualized form of associations between phenotype and genotype, in which SNPs are ordered based on their chromosome and base-pair positions. In a Manhattan plot, the $x$-axis thus represents the genomic position of each SNP and $y$-axis represents the negative logarithm of the $P$-value generated from the F-test for testing $\mathrm{H}_{0}$. Here, both heat map and Manhattan plots were obtained from an enhanced comparison scenario using the GAPIT package [64].

\section{Gene annotation}

Sequences surrounding all significantly associated SNPs were obtained from the wheat $90 \mathrm{~K}$ SNP database [65] used for assessing gene annotation using Gramene (http://www.gramene.org/) by aligning them to the IWGSC RefSeq v1.0 annotation (https://wheat-urgi.versailles.inra.fr/Seq-Repository/Annotations). The function of putative genes was explored by investigating the pathways which the encoded enzymes were involved in. After aligning SNPs sequences to the reference genome, overlapping genes with the highest identity percentage and blast score were selected for further processing. The gene ontology of each selected gene, including molecular function and biological process, were extracted from the ensemble-gramene database (http://ensembl.gramene. org).

\section{Phenotyping data analysis and calculation of favorable allele effect}

Phenotypic data were analyzed using SAS v.9.4 [66] separately for the two environments. The adjusted means were then obtained from the alpha-lattice design used for advanced linear analysis. Adjusted means were estimated using GLM and Mixed procedures. The phenotypic effect of favorable alleles (ai) was estimated using the following formula:

$$
a i=\left(\frac{\Sigma x_{i j}}{n_{i}}\right)-\left(\frac{\Sigma N_{K}}{n_{K}}\right)
$$

where, $x_{i j}$ is the phenotypic value of the $j$ th individual for the $i$ th allele, $n_{i}$ is the number of individuals carrying the $j$ th allele, $N_{K}$ is the nth individual phenotypic value for all entries, and $n_{K}$ is the number of individuals. Positive and negative effects of all alleles are represented by $a i>0$, and $a i<0$, respectively.

\section{Supplementary information}

Supplementary information accompanies this paper at https://doi.org/10. 1186/s12870-019-2165-4

Additional file $\mathbf{1}$ Table S1. The information of Iranian wheat accessions including varieties released between 1942 and 2014, and landraces collected between 1931 and 1968

Additional file 3 Fig. S1. Climate condition include precipitation and temperature of field trail site during the 2016-17 and 2017-18 cropping seasons. Fig. S2. The pattern of LD decay in different chromosomes of genome $A$ in $T$. aestivum based on original SNPS. Fig. S3. The pattern of LD decay in different chromosomes of genome $B$ in $T$. aestivum based on original SNPs. Fig. S4. The pattern of LD decay in different chromosomes of genome D in T. aestivum based on original SNPS.

Additional file $\mathbf{2}$ Table S2. Analysis of variance for agronomic traits of Iranian wheat accessions exposed to the well-watered conditions over two years. Table S3. Analysis of variance for agronomic traits of Iranian wheat accessions exposed to the rain-fed conditions over two years. Table S4. Person's coefficient of correlation between agronomic traits of Iranian wheat accessions over two years under well-watered conditions. Table S5. Pearson's confidents of correlation between agronomic traits of Iranian bread wheat accessions over two years under rain-fed conditions.

Additional file $\mathbf{1}$ Table S1. The information of Iranian wheat accessions including varieties released between 1942 and 2014, and landraces collected between 1931 and 1968

Additional file $3 \mathbf{~ F i g . ~ S 1 . ~ C l i m a t e ~ c o n d i t i o n ~ i n c l u d e ~ p r e c i p i t a t i o n ~ a n d ~}$ temperature of field trail site during the 2016-17 and 2017-18 cropping seasons. Fig. S2. The pattern of LD decay in different chromosomes of genome $A$ in $T$. aestivum based on original SNPs. Fig. S3. The pattern of LD decay in different chromosomes of genome $B$ in $T$. aestivum based on original SNPs. Fig. S4. The pattern of LD decay in different chromosomes of genome $D$ in $T$. aestivum based on original SNPS.

Additional file $\mathbf{4}$ Table S6. Description of expected MTAs using original SNPs for agronomic traits of Iranian wheat accessions exposed to the well-watered condition. Table S7. Description of expected MTAs using original SNPs for agronomic traits of Iranian wheat accessions exposed to the rain-fed condition. Table S8. Description of expected MTAs using imputed SNPs for agronomic traits of Iranian wheat accessions exposed to the well-watered condition. Table S9. Description of expected MTAs using imputed SNPs for agronomic traits of Iranian wheat accessions exposed to the rain-fed condition.

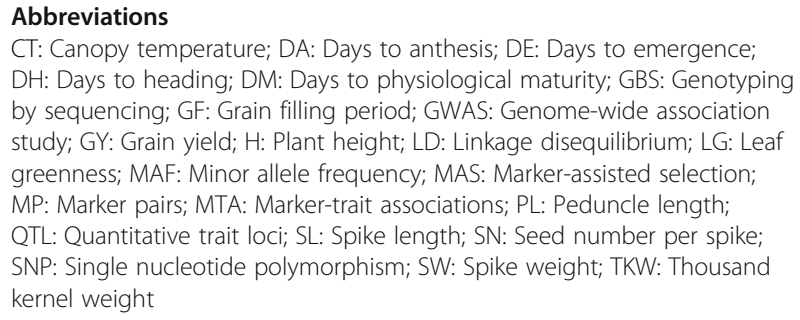
greenness; MAF: Minor allele frequency; MAS: Marker-assisted selection; MP: Marker pairs; MTA: Marker-trait associations; PL: Peduncle length; QTL: Quantitative trait loci; SL: Spike length; SN: Seed number per spike; SNP: Single nucleotide polymorphism; SW: Spike weight; TKW: Thousand kernel weight

\section{Acknowledgments}

We are grateful to Dr. Thomas Richards from the Department of Ecology and Genetics, Uppsala University for his advice and revising the manuscript.

\section{Authors' contributions}

YR: Performed field trial, analyzed the datasets, and wrote the manuscript. MRB: Assistance in data analysis and writing the manuscript. AT: Assistance in data analysis and revising the manuscript. HA and PKI: Assistance in data analysis and writing the manuscript. All authors contributed to revising and editing the manuscript. All authors have read and approved of the final manuscript. 


\section{Funding}

This work was supported by the University of Tehran and Iran National Science Foundation (grant no. 96008453). The funding body was involved in the material creation, designing the study, data analysis and writing the manuscript.

\section{Availability of data and materials}

The datasets generated and analysed during the current study are available in Figshare. SNP data files for 5, 6, 7, and 8 runs can be each accessed at: https://figshare.com/s/92cd9f23d9dddb224304;

https://figshare.com/s/b402031d86d969a7cb0f; https://figshare.com/s/83b4abe205a8a5409017;

https://figshare.com/s/253e39735d226b78b6bd.

\section{Ethics approval and consent to participate}

Not applicable.

\section{Consent for publication}

Not applicable.

\section{Competing interests}

The authors declare that they have no competing interests. The funding body was involved in the material creation, designing the study, data analysis and writing the manuscript.

\section{Author details}

'Department of Agronomy and Plant Breeding, Faculty of Agriculture, University of Tehran, Karaj, Iran. ${ }^{2}$ Department of Plant Breeding and Biotechnology, Faculty of Agriculture, Urmia University, Urmia, Iran. ${ }^{3}$ Linnean Centre for Plant Biology, Department of Plant Biology, Swedish University of Agricultural Sciences, Uppsala, Sweden.

Received: 18 June 2019 Accepted: 26 November 2019 Published online: 05 December 2019

\section{References}

1. Curtis T, Halford NG. Food security: the challenge of increasing wheat yield and the importance of not compromising food safety. Ann Appl Biol. 2014; 164(3):354-72

2. Tilman D, Cassman KG, Matson PA, Naylor R, Polasky S. Agricultural sustainability and intensive production practices. Nature. 2002;418(6898): 671-7.

3. O'Leary GJ, Christy B, Nuttall J, Huth N, Cammarano D, Stöckle C, Basso B, Shcherbak I, Fitzgerald G, Luo Q. Response of wheat growth, grain yield and water use to elevated $\mathrm{CO}_{2}$ under a free-air $\mathrm{CO}_{2}$ enrichment (FACE) experiment and modelling in a semi-arid environment. Glob Chang Biol. 2015;21(7):2670-86.

4. Richards RA, Hunt JR, Kirkegaard JA, Passioura JB. Yield improvement and adaptation of wheat to water-limited environments in Australia-a case study. Crop Pasture Sci. 2014:65(7):676-89.

5. Initiative AG. Analysis of the genome sequence of the flowering plant Arabidopsis thaliana. Nature. 2000:408(6814):796-815.

6. Goff SA, Ricke D, Lan T-H, Presting G, Wang R, Dunn M, Glazebrook J, Sessions A, Oeller $P$, Varma $H$, et al. A draft sequence of the rice genome (Oryza sativa L. ssp. japonica). Science. 2002;296(5565):92-100.

7. Beier S, Himmelbach A, Colmsee C, Zhang X-Q, Barrero RA, Zhang Q, Li L, Bayer M, Bolser D, Taudien S, et al. Construction of a map-based reference genome sequence for barley. Hordeum vulgare L Scientific Data. 2017;4 170044.

8. Mayer KFX, Rogers J, Dolezel J, Pozniak C, Eversole K, Feuillet C, Gill B, Friebe B, Lukaszewski AJ, Sourdille $P$, et al. A chromosome-based draft sequence of the hexaploid bread wheat (Triticum aestivum) genome. Science. 2014; 345(6194).

9. Brondani C, Rangel PHN, Brondani RPV, Ferreira ME. QTL mapping and introgression of yield-related traits from Oryza glumaepatula to cultivated rice (Oryza sativa) using microsatellite markers. Theor Appl Genet. 2002; 104(6-7):1192-203.

10. Buerstmayr H, Ban T, Anderson JA. QTL mapping and marker-assisted selection for Fusarium head blight resistance in wheat: a review. Plant Breed. 2009:128(1):1-26.
11. Collard BCY, Jahufer MZZ, Brouwer JB, Pang ECK. An introduction to markers, quantitative trait loci (QTL) mapping and marker-assisted selection for crop improvement: the basic concepts. Euphytica. 2005;142(1-2):169-96.

12. Huang C, Nie X, Shen C, You C, Li W, Zhao W, Zhang X, Lin Z. Population structure and genetic basis of the agronomic traits of upland cotton in China revealed by a genome-wide association study using high-density SNPs. Plant Biotechnol J. 2017;15(11):1374-86.

13. Saidou A-A, Thuillet A-C, Couderc M, Mariac C, Vigouroux Y. Association studies including genotype by environment interactions: prospects and limits. BMC Genet. 2014;15(1):3.

14. Neumann K, Kobiljski B, Dencic S, Varshney RK, Boerner A. Genome-wide association mapping: a case study in bread wheat (Triticum aestivum L.). Mol Breed. 2011:27(1):37-58.

15. Huang X, Wei X, Sang T, Zhao Q, Feng Q, Zhao Y, Li C, Zhu C, Lu T, Zhang $Z$, et al. Genome-wide association studies of 14 agronomic traits in rice landraces. Nat Genet. 2010;42(11):961-76.

16. Pasam RK, Sharma R, Malosetti M, van Eeuwijk FA, Haseneyer G, Kilian B, Graner A. Genome-wide association studies for agronomical traits in a world wide spring barley collection. BMC Plant Biol. 2012;12:16.

17. Li H, Peng Z, Yang X, Wang W, Fu J, Wang J, Han Y, Chai Y, Guo T, Yang N, et al. Genome-wide association study dissects the genetic architecture of oil biosynthesis in maize kernels. Nat Genet. 2013;45(1):43-50.

18. Sukumaran S, Dreisigacker S, Lopes M, Chavez P, Reynolds MP. Genomewide association study for grain yield and related traits in an elite spring wheat population grown in temperate irrigated environments. Theor Appl Genet. 2015;128(2):353-63.

19. Bordes J, Ravel C, Jaubertie JP, Duperrier B, Gardet O, Heumez E, Pissavy AL, Charmet G, Le Gouis J, Balfourier F. Genomic regions associated with the nitrogen limitation response revealed in a global wheat core collection. Theor Appl Genet. 2013;126(3):805-22.

20. Zanke C, Ling J, Plieske J, Kollers S, Ebmeyer E, Korzun V, Argillier O, Stiewe $G$, Hinze M, Beier $S$, et al. Genetic architecture of main effect QTL for heading date in European winter wheat. Front Plant Sci. 2014;5:217.

21. Q-u A, Rasheed A, Anwar A, Mahmood T, Imtiaz M, Mahmood T, Xia X, He Z, Quraishi UM. Genome-wide association for grain yield under rainfed conditions in historical wheat cultivars from Pakistan. Front Plant Sci. 2015;6: 743.

22. Elliott LT, Sharp K, Alfaro-Almagro F, Shi S, Miller KL, Douaud G, Marchini J, Smith SM. Genome-wide association studies of brain imaging phenotypes in UK biobank. Nature. 2018;562(7726):210-6.

23. Khera AV, Chaffin M, Aragam KG, Haas ME, Roselli C, Choi SH, Natarajan P, Lander ES, Lubitz SA, Ellinor PT, et al. Genome-wide polygenic scores for common diseases identify individuals with risk equivalent to monogenic mutations. Nat Genet. 2018;50(9):1219-24.

24. Marchini J, Howie B. Genotype imputation for genome-wide association studies. Nat Rev Genet. 2010;11(7):499-511.

25. He S, Zhao Y, Mette MF, Bothe R, Ebmeyer E, Sharbel TF, Reif JC, Jiang Y Prospects and limits of marker imputation in quantitative genetic studies in European elite wheat (Triticum aestivum L.). BMC genomics. 2015;16(1):168.

26. Fu Y-B. Genetic diversity analysis of highly incomplete SNP genotype data with imputations: an empirical assessment. G3: Genes Genomes Genetics. 2014;4(5):891-900

27. Li Y, Willer C, Sanna S, Abecasis G. Genotype imputation. Annu Rev Genomics Hum Genet. 2009;10:387-406.

28. Nyine M, Wang S, Kiani K, Jordan K, Liu S, Byrne P, Haley S, Baenziger S, Chao S, Bowden R, et al. Genotype imputation in winter wheat using firstgeneration haplotype map SNPs improves genome-wide association mapping and genomic prediction of traits. G3-Genes Genomes Genetics. 2019;9(1):125-33.

29. Subirana I, Gonzalez JR. Genetic association analysis and meta-analysis of imputed SNPs in longitudinal studies. Genet Epidemiol. 2013;37(5):465-77.

30. Alipour H, Bihamta MR, Mohammadi V, Peyghambari SA, Bai G, Zhang G. Genotyping-by-sequencing (GBS) revealed molecular genetic diversity of iranian wheat landraces and cultivars. Front Plant Sci. 2017:8:1293.

31. Alipour H, Bai G, Zhang G, Bihamta MR, Mohammadi V, Peyghambari SA. Imputation accuracy of wheat genotyping-by-sequencing (GBS) data using barley and wheat genome references. PLoS One. 2019;14(1):e0208614.

32. Berkman PJ, Visendi P, Lee HC, Stiller J, Manoli S, Lorenc MT, Lai K, Batley J, Fleury D, Simkova $\mathrm{H}$, et al. Dispersion and domestication shaped the genome of bread wheat. Plant Biotechnol J. 2013;11(5):564-71. 
33. Edae EA, Bowden RL, Poland J. Application of population sequencing (POPSEQ) for ordering and imputing genotyping-by-sequencing markers in hexaploid wheat. G3-Genes Genomes Genetics. 2015;5(12):2547-53.

34. Dvorak J, Akhunov ED, Akhunov AR, Deal KR, Luo M-C. Molecular characterization of a diagnostic DNA marker for domesticated tetraploid wheat provides evidence for gene flow from wild tetraploid wheat to hexaploid wheat. Mol Biol Evol. 2006;23(7):1386-96.

35. Jordan KW, Wang S, Lun Y, Gardiner L-J, MacLachlan R, Hucl P, Wiebe K, Wong D, Forrest KL, Sharpe AG, Consortium IWGS. A haplotype map of allohexaploid wheat reveals distinct patterns of selection on homoeologous genomes. Genome Biol. 2015;16:48.

36. Chao S, Zhang W, Akhunov E, Sherman J, Ma Y, Luo M-C, Dubcovsky J. Analysis of gene-derived SNP marker polymorphism in US wheat (Triticum aestivum L.) cultivars. Mol Breed. 2009;23(1):23-33.

37. Gaut BS, Long AD. The lowdown on linkage disequilibrium. Plant Cell. 2003; 15(7):1502-6.

38. Liu H, Zhou H, Wu Y, Li X, Zhao J, Zuo T, Zhang X, Zhang Y, Liu S, Shen Y, et al. The impact of genetic relationship and linkage disequilibrium on genomic selection. PLoS One. 2015;10(7):e0132379.

39. Yan J, Shah T, Warburton ML, Buckler ES, McMullen MD, Crouch J. Genetic characterization and linkage disequilibrium estimation of a global maize collection using SNP markers. PLoS One. 2009;4(12):e8451.

40. Abdullaev AA, Salakhutdinov IB, Egamberdiev SS, Khurshut EE, Rizaeva SM, Ulloa M, Abdurakhmonov IY. Genetic diversity, linkage disequilibrium, and association mapping analyses of Gossypium barbadense L. germplasm. PLOS ONE. 2017;12(11):e0188125

41. Oraguzie NC, Wilcox PL, Rikkerink EHA, de Silva HN. Linkage Disequilibrium. In: Oraguzie NC, EHA R, Gardiner SE, De Silva HN, editors. Association Mapping in Plants. New York, NY: Springer New York; 2007. p. 11-39.

42. Marza F, Bai GH, Carver BF, Zhou WC. Quantitative trait loci for yield and related traits in the wheat population Ning7840 x Clark. Theor Appl Genet. 2006;112(4):688-98.

43. Wang RX, Hai L, Zhang XY, You GX, Yan CS, Xiao SH. QTL mapping for grain filling rate and yield-related traits in RILs of the Chinese winter wheat population Heshangmai X Yu8679. Theor Appl Genet. 2009;118(2):313-25.

44. Groos C, Robert N, Bervas E, Charmet G. Genetic analysis of grain proteincontent, grain yield and thousand-kernel weight in bread wheat. Theor Appl Genet. 2003;106(6):1032-40.

45. Bossolini E, Wicker T, Knobel PA, Keller B. Comparison of orthologous loci from small grass genomes Brachypodium and rice: implications for wheat genomics and grass genome annotation. Plant J. 2007:49:704-17.

46. Acuna-Galindo MA, Mason RE, Subramanian NK, Hays DB. Meta-analysis of wheat QTL regions associated with adaptation to drought and heat stress. Crop Sci. 2015;55(2):477-92.

47. Lopes MS, Dreisigacker S, Pena RJ, Sukumaran S, Reynolds MP. Genetic characterization of the wheat association mapping initiative (WAMI) panel for dissection of complex traits in spring wheat. Theor Appl Genet. 2015; 128(3):453-64

48. Pinto RS, Reynolds MP, Mathews KL, Mclntyre CL, Olivares-Villegas J-J, Chapman SC. Heat and drought adaptive QTL in a wheat population designed to minimize confounding agronomic effects. Theor Appl Genet. 2010;121(6):1001-21.

49. Tuberosa R, Salvi S. Genomics-based approaches to improve drought tolerance of crops. Trends Plant Sci. 2006;11(8):405-12.

50. Cattivelli L, Rizza F, Badeck F-W, Mazzucotelli E, Mastrangelo AM, Francia E, Mare C, Tondelli A, Stanca AM. Drought tolerance improvement in crop plants: an integrated view from breeding to genomics. Field Crop Res. 2008; 105(1-2):1-14

51. Woldesemayat AA, Van Heusden P, Ndimba BK, Christoffels A. An integrated and comparative approach towards identification, characterization and functional annotation of candidate genes for drought tolerance in sorghum (Sorghum bicolor (L.) Moench). BMC Genet. 2017;18:119.

52. Sairam RK, Saxena DC. Oxidative stress and antioxidants in wheat genotypes: possible mechanism of water stress tolerance. J Agron Crop Sci. 2000;184(1):55-61

53. Antao CM, Malcata FX. Plant serine proteases: biochemical, physiological and molecular features. Plant Physiol Biochem. 2005;43(7):637-50.

54. Zhang H, Mao X, Wang C, Jing R. Overexpression of a common wheat gene TaSnRK2.8 enhances tolerance to drought, salt and low temperature in Arabidopsis. PLOS ONE. 2010;5(12):e16041.
55. Nguema-Ona E, Vicre-Gibouin M, Gotte M, Plancot B, Lerouge P, Bardor M, Driouich A. Cell wall O-glycoproteins and N-glycoproteins: aspects of biosynthesis and function. Front Plant Sci. 2014;5:499.

56. Caverzan A, Casassola A, Brammer SP. Antioxidant responses of wheat plants under stress. Genet Mol Biol. 2016;39(1):1-6.

57. Karacic Z, Vukelic B, Ho GH, Jozic I, Sucec I, Salopek-Sondi B, Kozlovic M, Brenner SE, Ludwig-Muller J, Abramic M. A novel plant enzyme with dual activity: an atypical Nudix hydrolase and a dipeptidyl peptidase III. Biol Chem. 2017;398(1):101-12.

58. Dragwidge JM, Scholl S, Schumacher K, Gendall AR. NHX-type Na+(K+)/H+ antiporters are required for TGN/EE trafficking and endosomal ion homeostasis in Arabidopsis thaliana. J Cell Sci. 2019;132(7):jcs226472.

59. Dong C, Wang J, Chen Q, Yu Y, Li B. Detection of favorable alleles for yield and yield components by association mapping in upland cotton. Genes \& Genomics. 2018;40(7):725-34

60. Browning BL, Browning SR. A unified approach to genotype imputation and haplotype-phase inference for large data sets of trios and unrelated individuals. Am J Hum Genet. 2009;84(2):210-23.

61. Team R: RStudio: integrated development for R. RStudio, Inc, Boston, MA URL http://www rstudio com 2015, 42:14.

62. Pritchard JK, Stephens M, Donnelly P. Inference of population structure using multilocus genotype data. Genetics. 2000;155(2):945-59.

63. Bradbury PJ, Zhang Z, Kroon DE, Casstevens TM, Ramdoss Y, Buckler ES. TASSEL: software for association mapping of complex traits in diverse samples. Bioinformatics. 2007;23(19):2633-5.

64. Lipka AE, Tian F, Wang Q, Peiffer J, Li M, Bradbury PJ, Gore MA, Buckler ES, Zhang Z. GAPIT: genome association and prediction integrated tool. Bioinformatics. 2012;28(18):2397-9.

65. Wang S, Wong D, Forrest K, Allen A, Chao S, Huang BE, Maccaferri M, Salvi S, Milner SG, Cattivelli L, et al. Characterization of polyploid wheat genomic diversity using a high-density 90000 single nucleotide polymorphism array. Plant Biotechnol J. 2014;12(6):787-96.

66. Institute S: Base SAS 9.4 procedures guide: Statistical procedures: SAS Institute; 2017

\section{Publisher's Note}

Springer Nature remains neutral with regard to jurisdictional claims in published maps and institutional affiliations.

Ready to submit your research? Choose BMC and benefit from:

- fast, convenient online submission

- thorough peer review by experienced researchers in your field

- rapid publication on acceptance

- support for research data, including large and complex data types

- gold Open Access which fosters wider collaboration and increased citations

- maximum visibility for your research: over $100 \mathrm{M}$ website views per year

At $\mathrm{BMC}$, research is always in progress.

Learn more biomedcentral.com/submissions 